\title{
Neo-Clerodane Diterpenoids and other Constituents of Salvia filipes
}

Emma Maldonado,* Leonel Galicia, Ma. Isabel Chávez and Simón Hernández-Ortega

Instituto de Química, Universidad Nacional Autónoma de México, Circuito Exterior, Ciudad Universitaria, Coyoacán, 04510, D. F., México.

All the NMR spectra were recorded on a Varian Inova $500\left({ }^{1} \mathrm{H}\right.$ at $500 \mathrm{MHz} ;{ }^{13} \mathrm{C}$ at $125 \mathrm{MHz}$, using TMS as internal standard $)$

Figure S1. ${ }^{1} \mathrm{H}$ NMR spectrum of compound $\mathbf{1}\left(\mathrm{CDCl}_{3}\right)$

Figure S2. ${ }^{13} \mathrm{C}$ NMR spectrum of compound $\mathbf{1}\left(\mathrm{CDCl}_{3}\right)$

Figure S3. COSY spectrum of compound $\mathbf{1}\left(\mathrm{CDCl}_{3}\right)$

Figure S4. HSQC spectrum of compound $1\left(\mathrm{CDCl}_{3}\right)$

Figure S5. $\mathrm{HMBC}$ spectrum of compound $\mathbf{1}\left(\mathrm{CDCl}_{3}\right)$

Figure S6. NOESY spectrum of compound $\mathbf{1}\left(\mathrm{CDCl}_{3}\right)$

Figure S7. ${ }^{1} \mathrm{H}$ NMR spectrum of compound $\mathbf{3}\left(\right.$ DMSO- $\left._{6}\right)$

Figure S8 ${ }^{13} \mathrm{C}$ NMR spectrum of compound $\mathbf{3}$ (DMSO-d $\mathrm{d}_{6}$ )

Figure S9. COSY spectrum of compound $\mathbf{3}\left(\mathrm{DMSO}_{-} \mathrm{d}_{6}\right)$

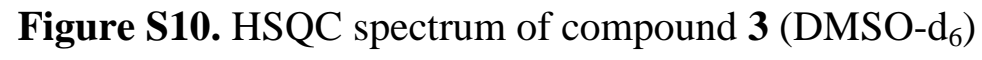

Figure S11. HMBC spectrum of compound $\mathbf{3}\left(\right.$ DMSO-d $\left._{6}\right)$

Figure S12. NOESY spectrum of compound $3\left(\right.$ DMSO-d $\left._{6}\right)$ 
Figure S13. ${ }^{1} \mathrm{H}$ NMR spectrum of compound 4/5 $\left(\mathrm{CDCl}_{3}\right)$

Figure S14. ${ }^{13} \mathrm{C}$ NMR spectrum of compound $4 / 5\left(\mathrm{CDCl}_{3}\right)$

Figure S15. COSY spectrum of compound $\mathbf{4 / 5}\left(\mathrm{CDCl}_{3}\right)$

Figure S16. HSQC spectrum of compound $4 / 5\left(\mathrm{CDCl}_{3}\right)$

Figure S17. HMBC spectrum of compound 4/5 $\left(\mathrm{CDCl}_{3}\right)$

Figure S18. NOESY spectrum of compound 4/5 $\left(\mathrm{CDCl}_{3}\right)$

Figure S19. ${ }^{1} \mathrm{H}$ NMR spectrum of compound 7 (DMSO- $\left.\mathrm{d}_{6}\right)$

Figure $\mathbf{S 2 0}{ }^{13} \mathrm{C}$ NMR spectrum of compound 7 (DMSO- $\mathrm{d}_{6}$ )

Figure S21. COSY spectrum of compound $7\left(\mathrm{DMSO}^{\left.-\mathrm{d}_{6}\right)}\right.$

Figure S22. HSQC spectrum of compound 7 (DMSO-d $\left.\mathrm{d}_{6}\right)$

Figure S23. HMBC spectrum of compound 7 (DMSO-d $\left.\mathrm{d}_{6}\right)$

Figure S24. NOESY spectrum of compound 7 (DMSO-d ${ }_{6}$ )

Figure S25. ${ }^{1} \mathrm{H}$ NMR spectrum of compound 8 (DMSO- $\left.\mathrm{d}_{6}\right)$

Figure S26 ${ }^{13} \mathrm{C}$ NMR spectrum of compound 8 (DMSO-d $\left.\mathrm{d}_{6}\right)$

Figure S27. COSY spectrum of compound 8 (DMSO- $\left.\mathrm{d}_{6}\right)$

Figure S28. HSQC spectrum of compound $8\left(D^{D M S O}-\mathrm{d}_{6}\right)$

Figure S29. HMBC spectrum of compound $8\left(\mathrm{DMSO}_{\mathrm{d}}\right)$

Figure S30. NOESY spectrum of compound $8\left(\mathrm{DMSO}_{-} \mathrm{d}_{6}\right)$

Table S1. ${ }^{1} \mathrm{H}$ and ${ }^{13} \mathrm{C}$ NMR Data of Compound $\mathbf{1 0}$ 


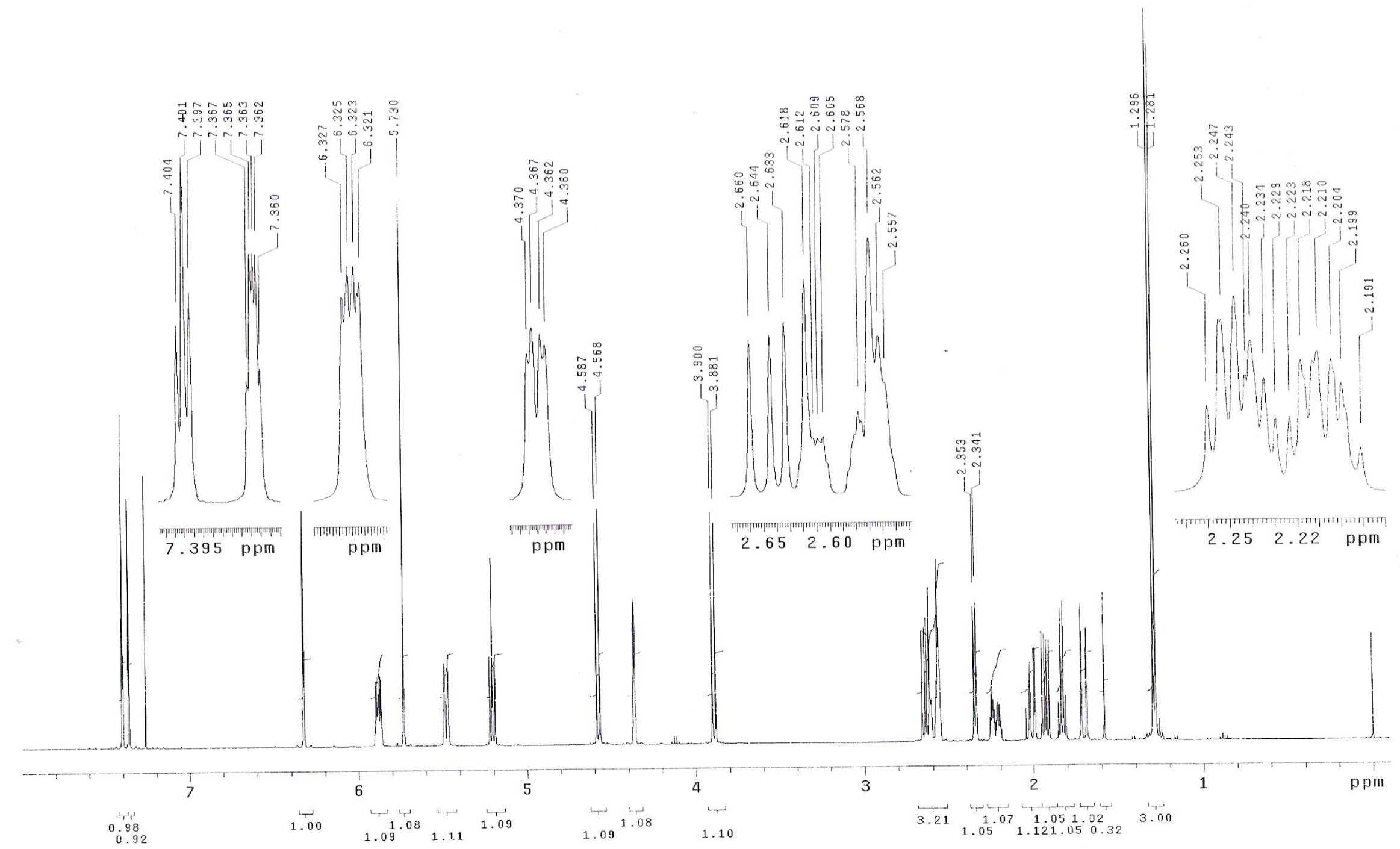

Figure S1. ${ }^{1} \mathrm{H}$ NMR spectrum of compound $\mathbf{1}\left(\mathrm{CDCl}_{3}\right)$ 


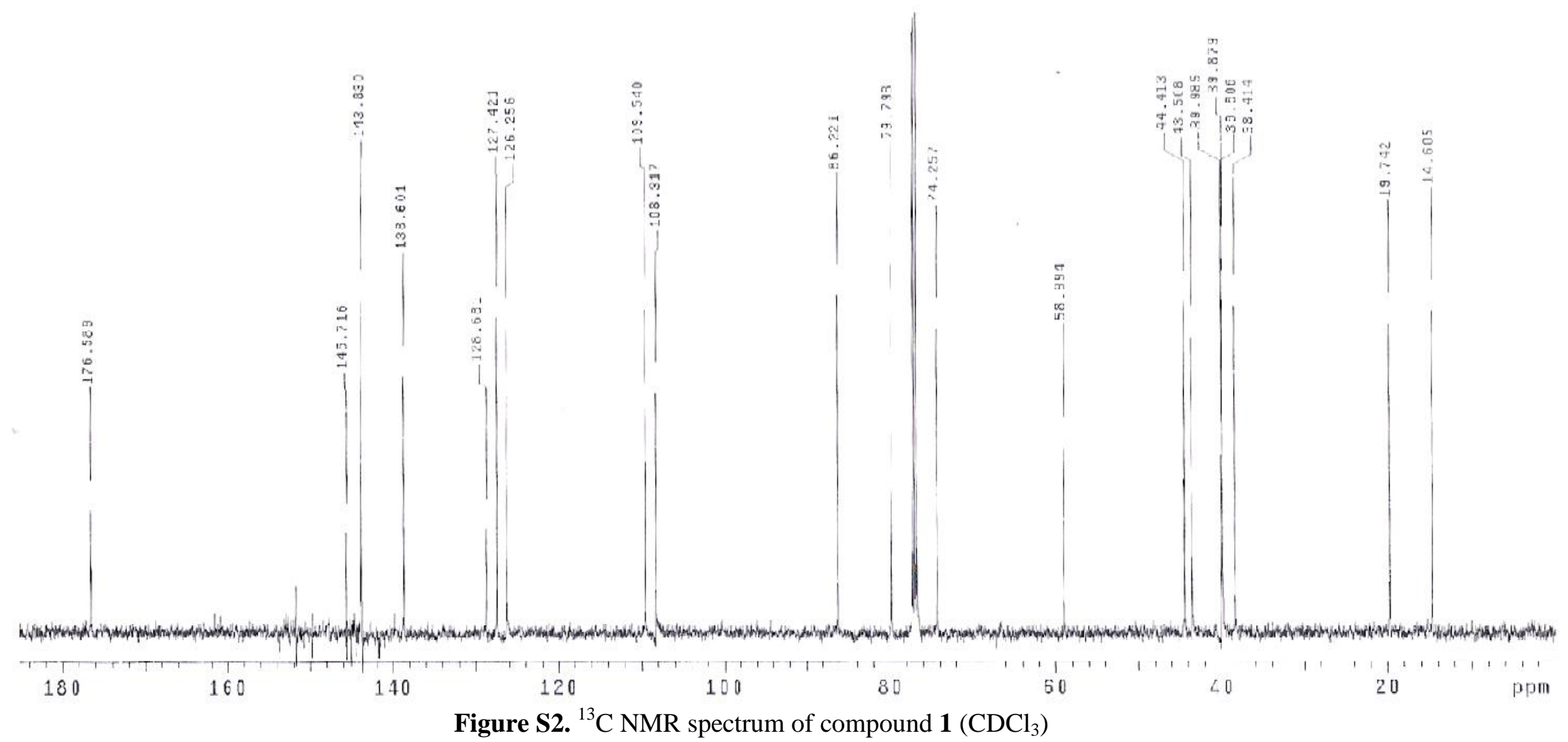




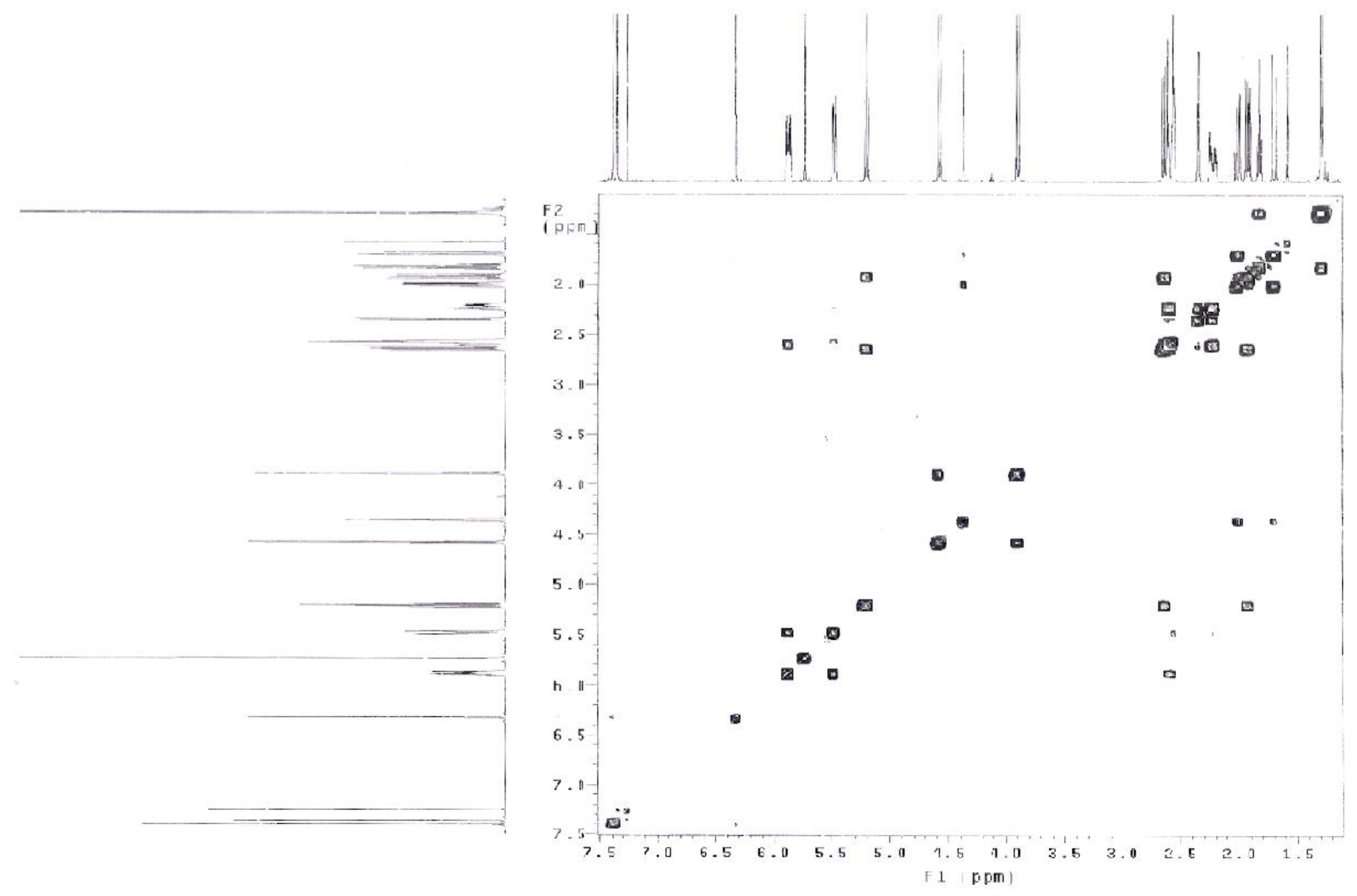

Figure S3. COSY spectrum of compound $\mathbf{1}\left(\mathrm{CDCl}_{3}\right)$ 


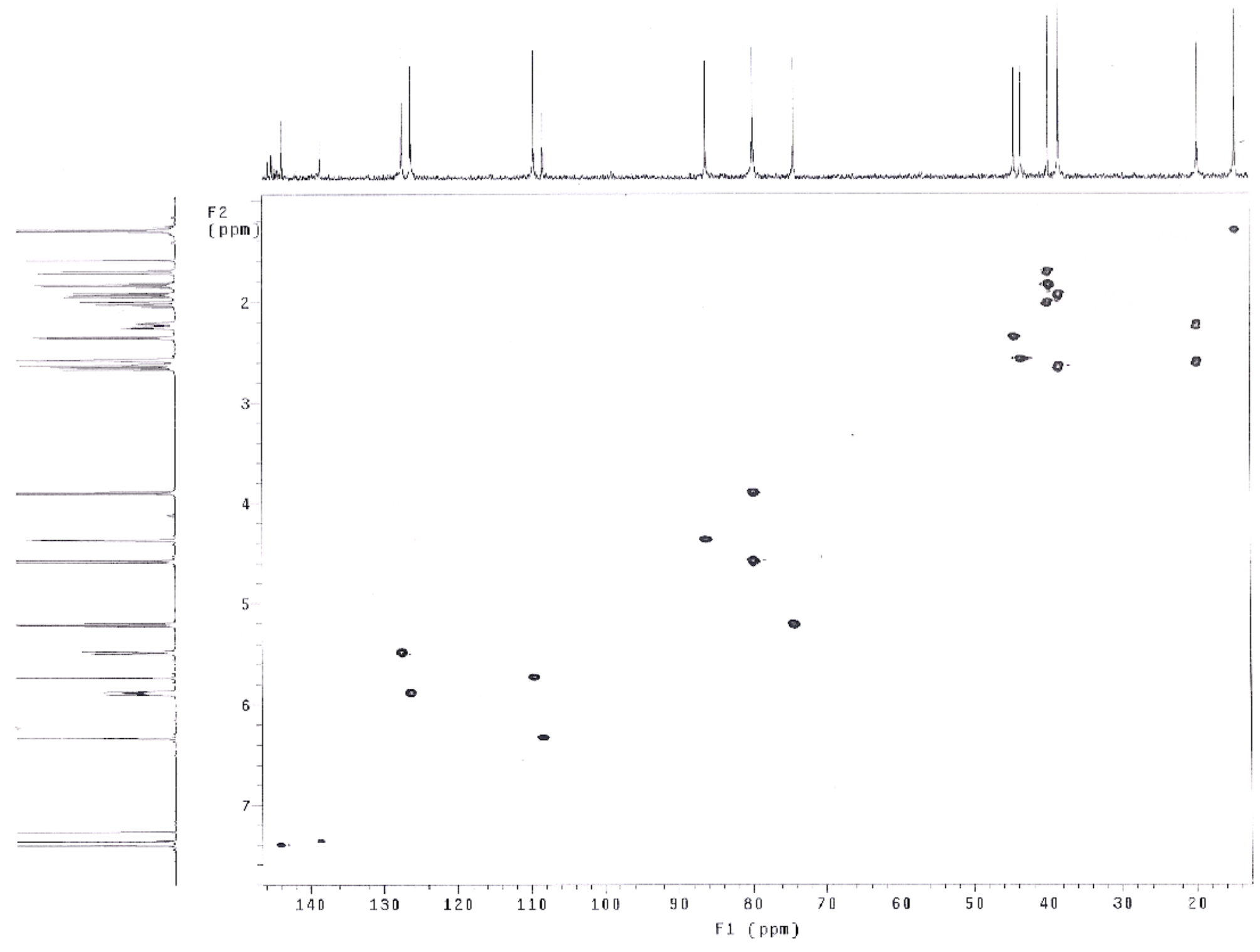

Figure S4. HSQC spectrum of compound $1\left(\mathrm{CDCl}_{3}\right)$ 


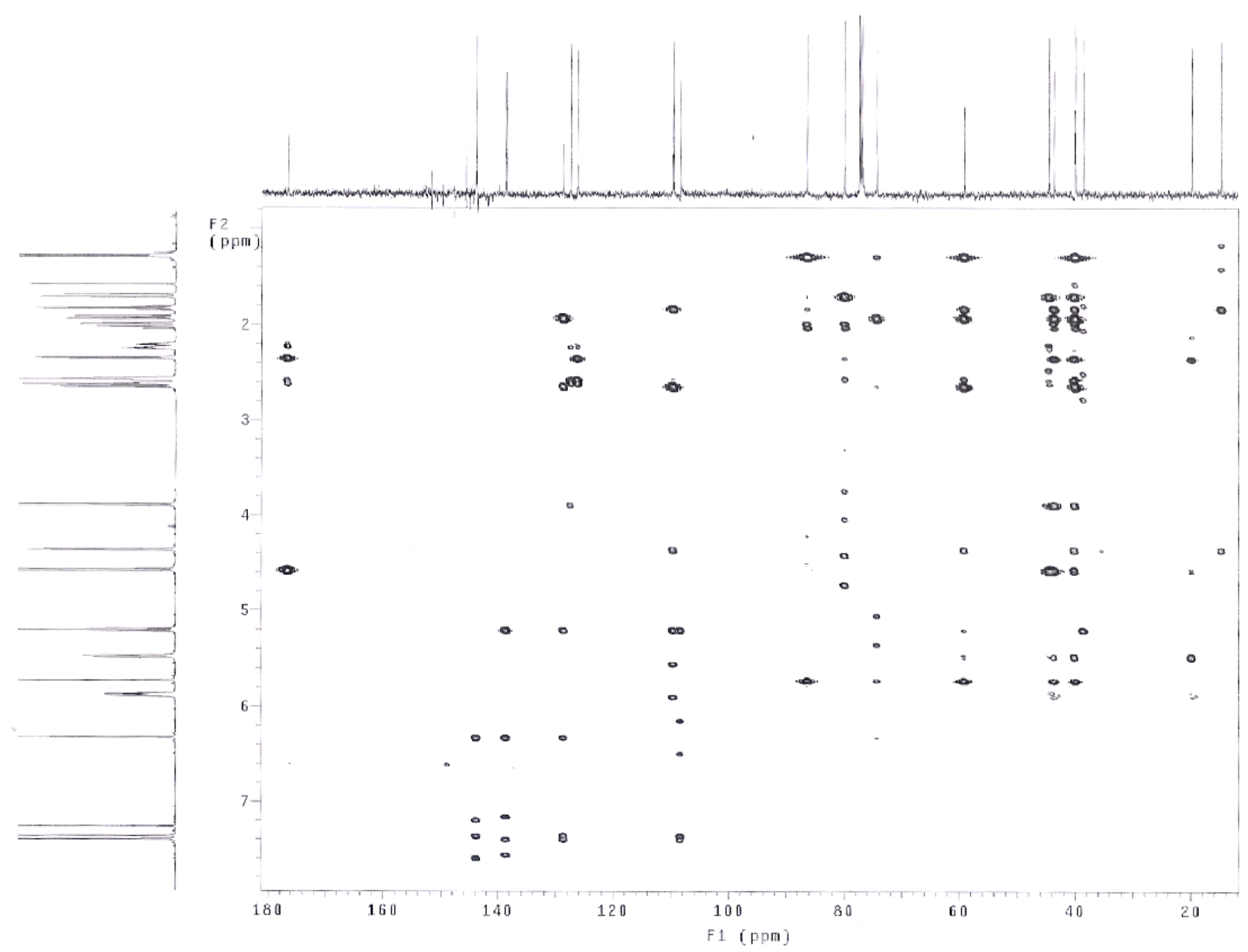

Figure S5. $\mathrm{HMBC}$ spectrum of compound $\mathbf{1}\left(\mathrm{CDCl}_{3}\right)$ 


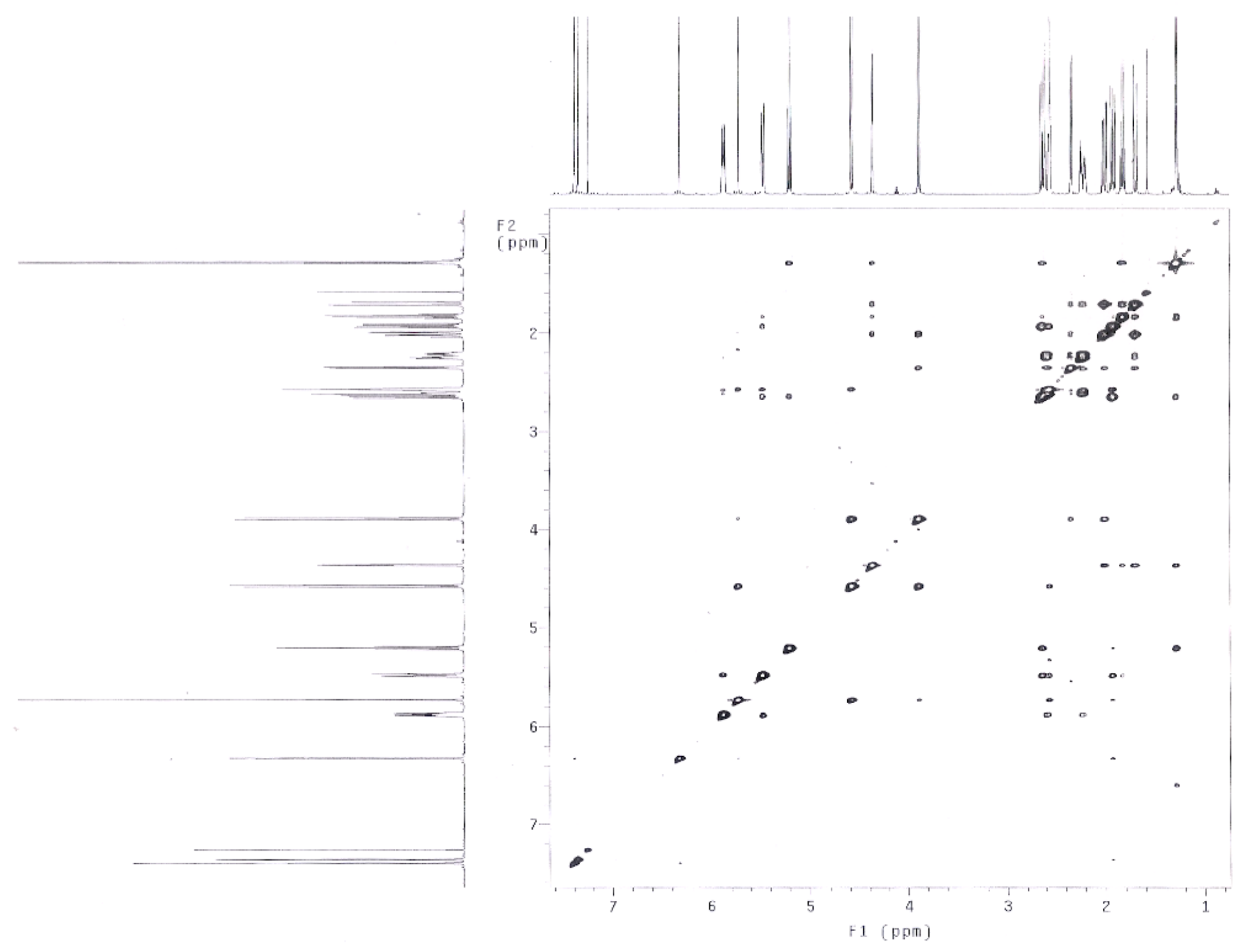

Figure S6. NOESY spectrum of compound $\mathbf{1}\left(\mathrm{CDCl}_{3}\right)$ 


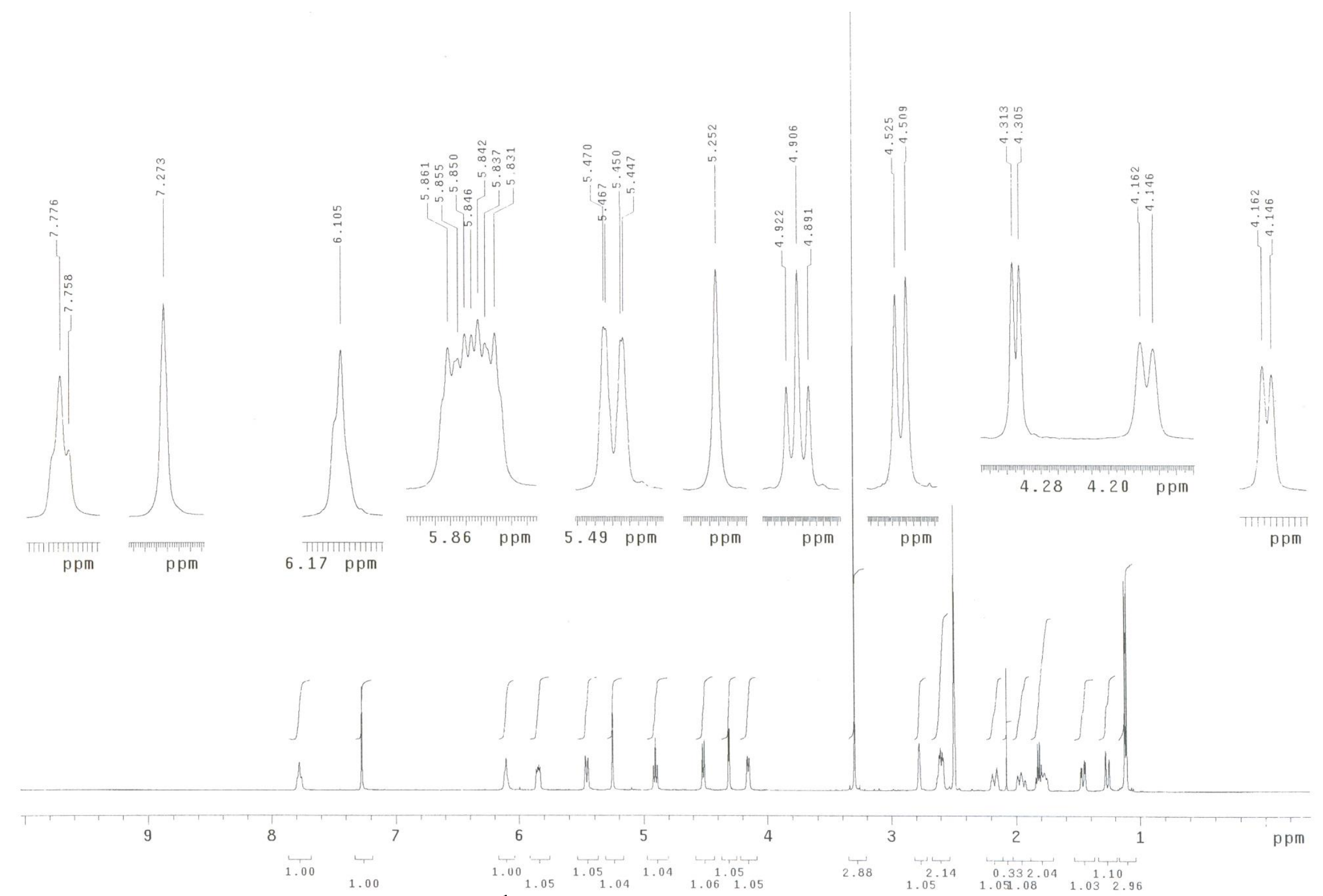

Figure S7. ${ }^{1} \mathrm{H}$ NMR spectrum of compound $3\left(\right.$ DMSO-d $\left._{6}\right)$ 


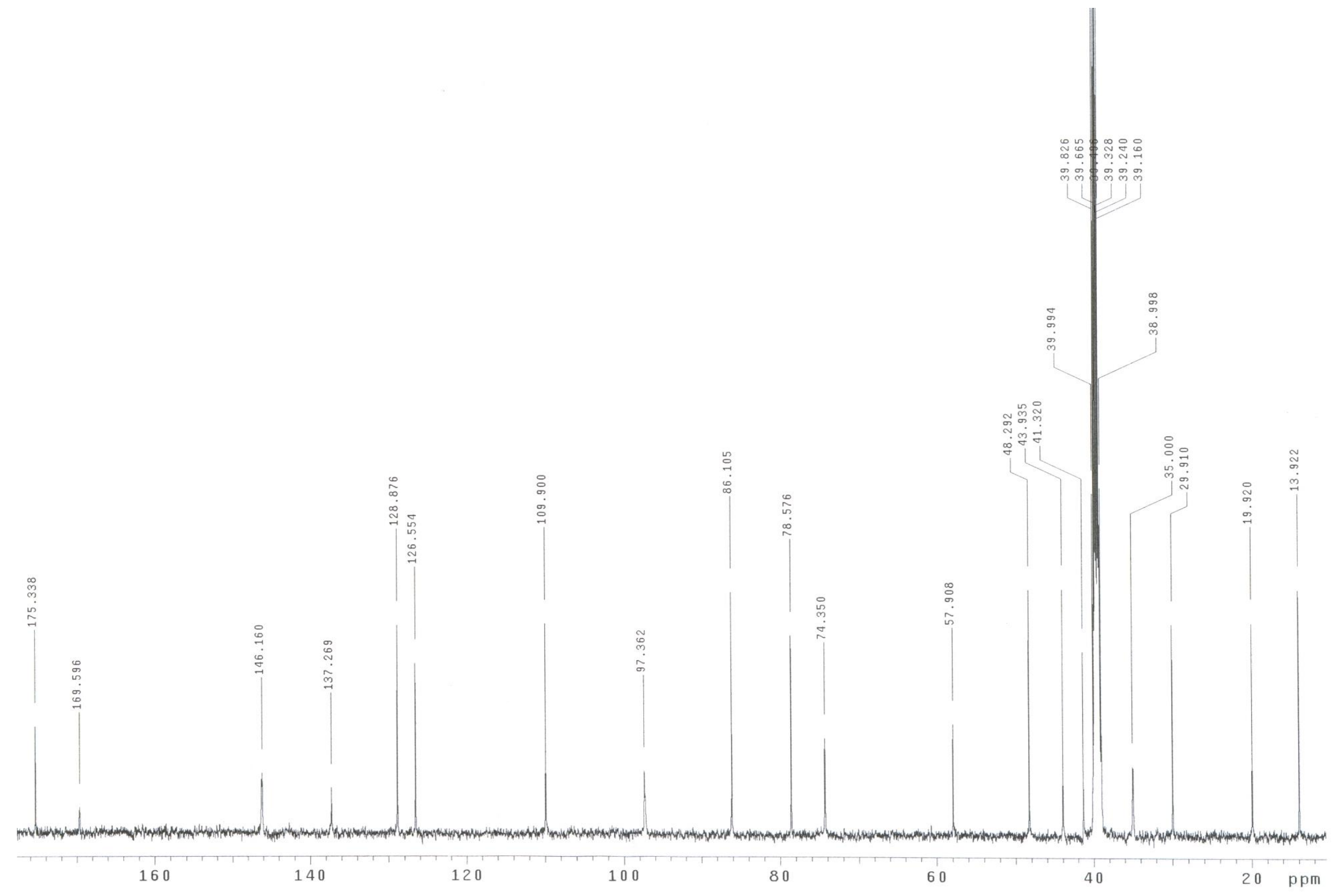

Figure S8 ${ }^{13} \mathrm{C}$ NMR spectrum of compound $3\left(\right.$ DMSO-d $\left._{6}\right)$ 

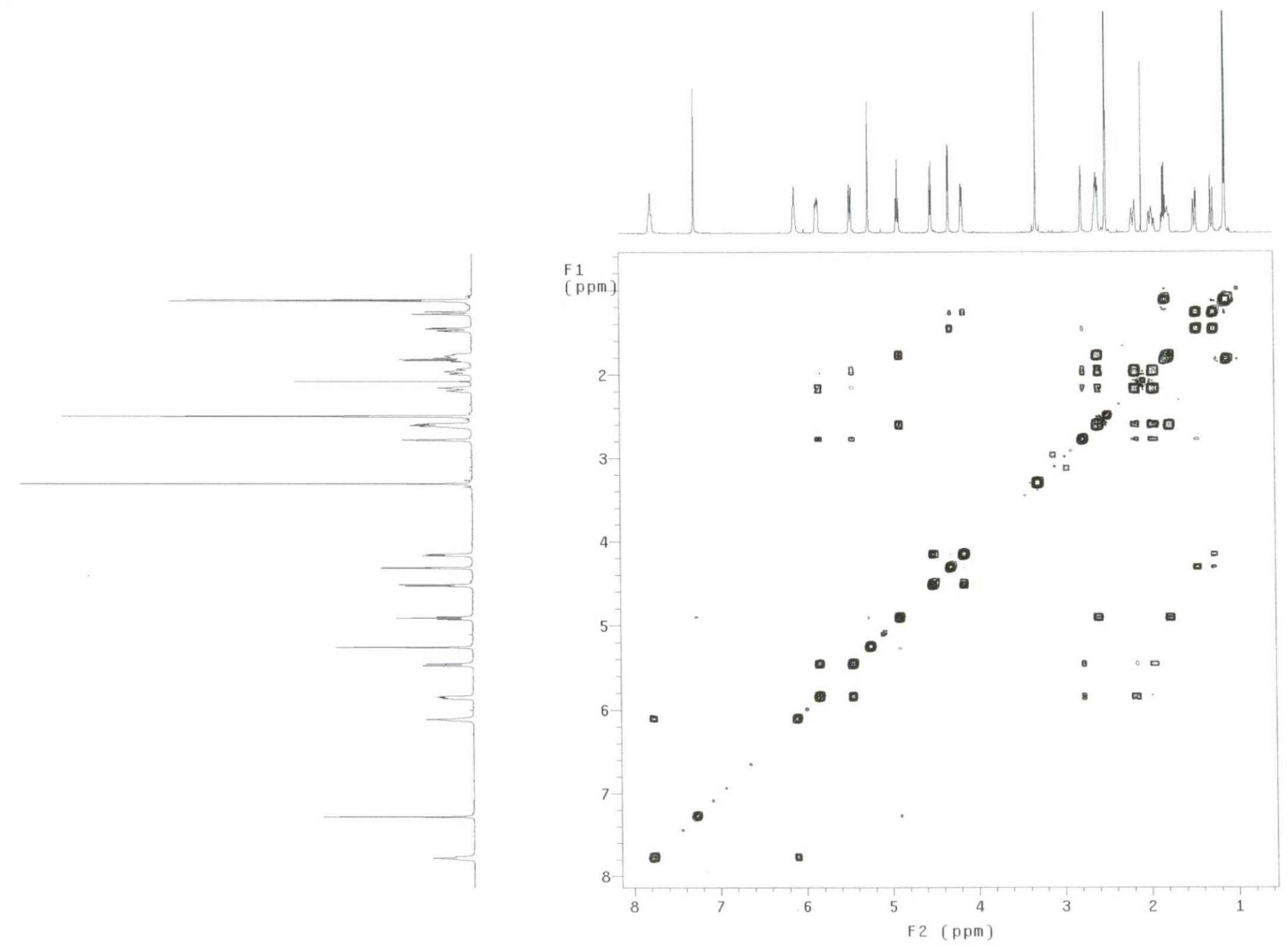

Figure S9. COSY spectrum of compound $3\left(D^{D M S O}-\mathrm{d}_{6}\right)$ 


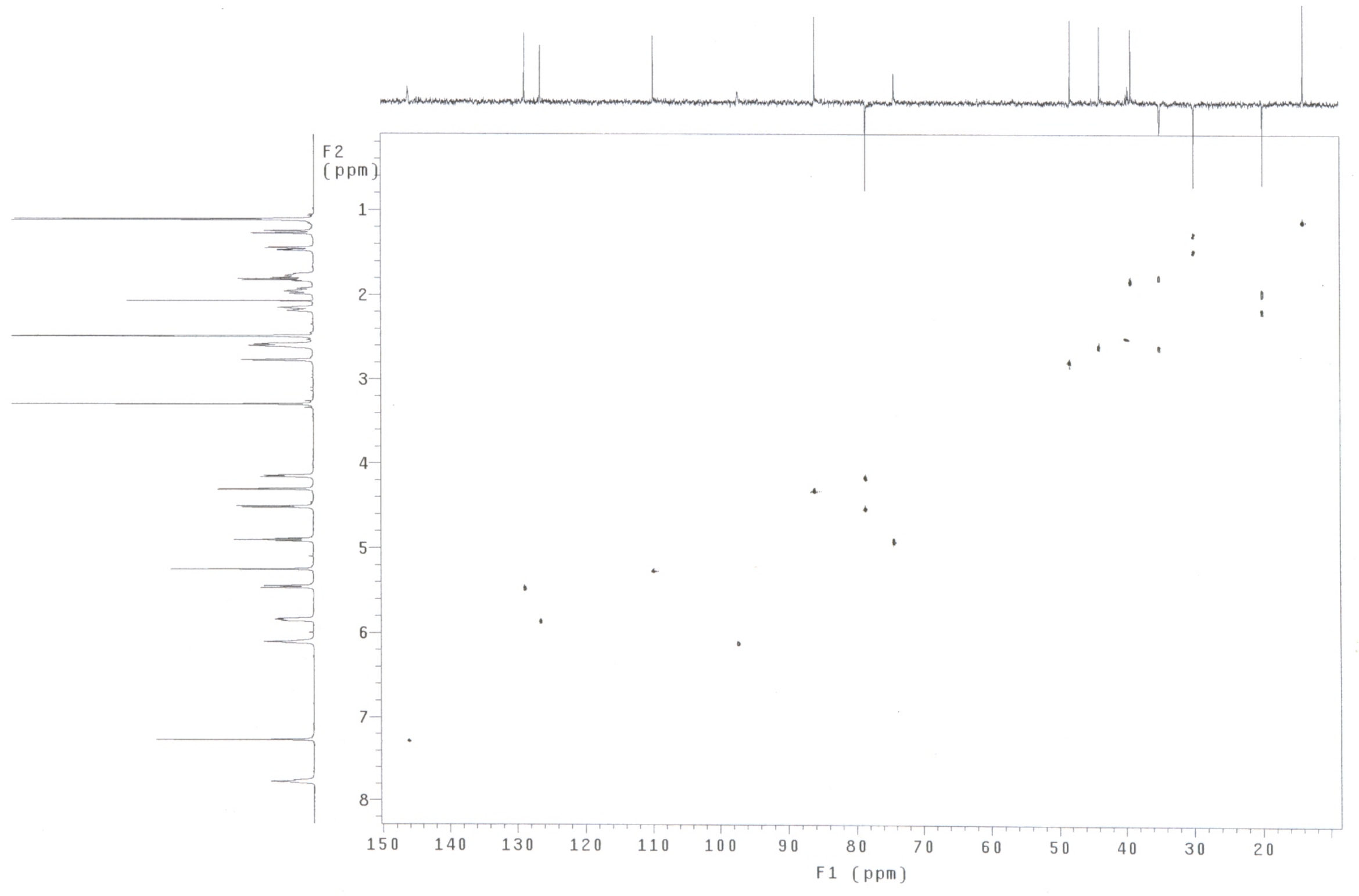

Figure S10. HSQC spectrum of compound $\mathbf{3}\left(\right.$ DMSO- $\left._{6}\right)$ 


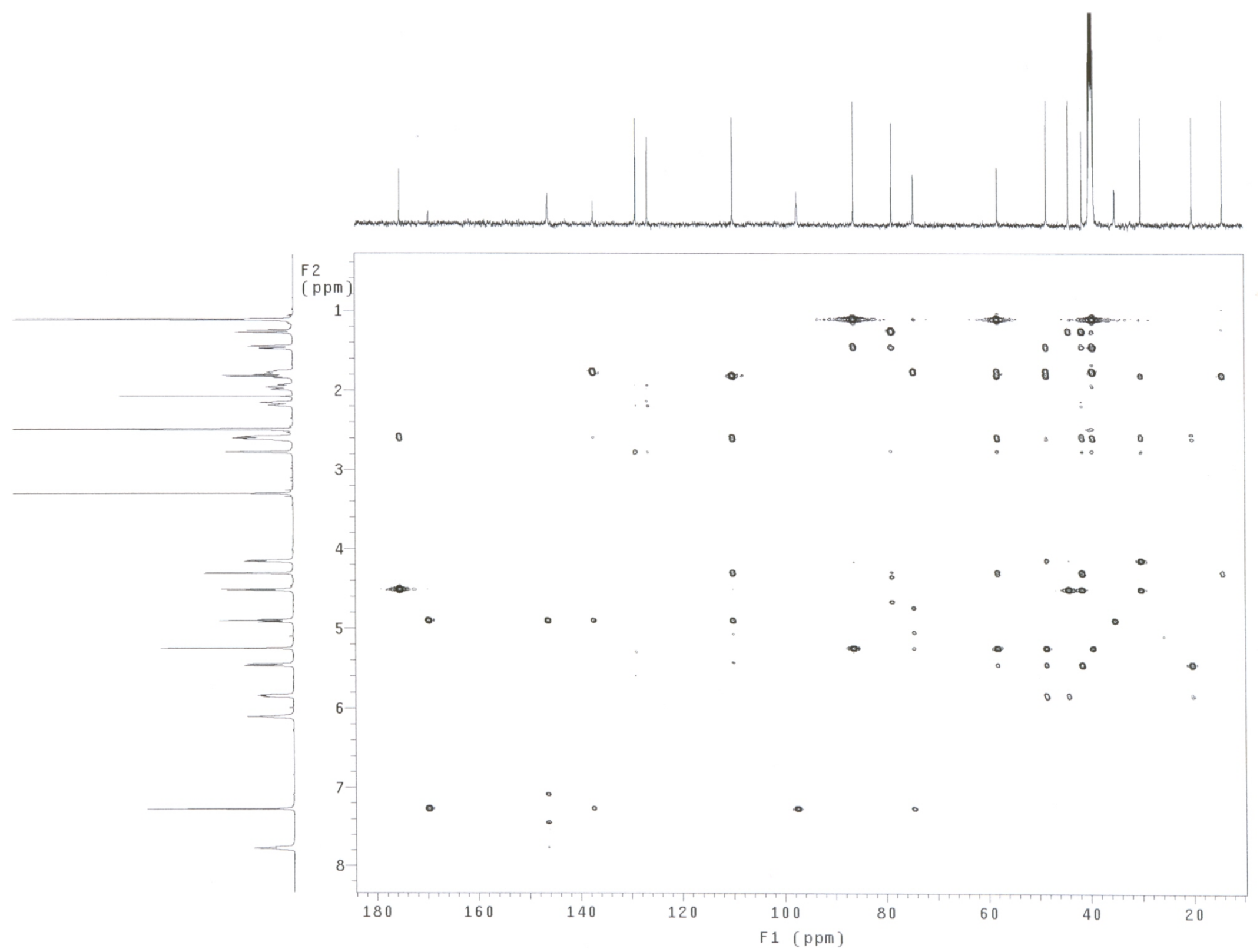

Figure S11. HMBC spectrum of compound $\mathbf{3}\left(\mathrm{DMSO}^{-\mathrm{d}_{6}}\right)$ 


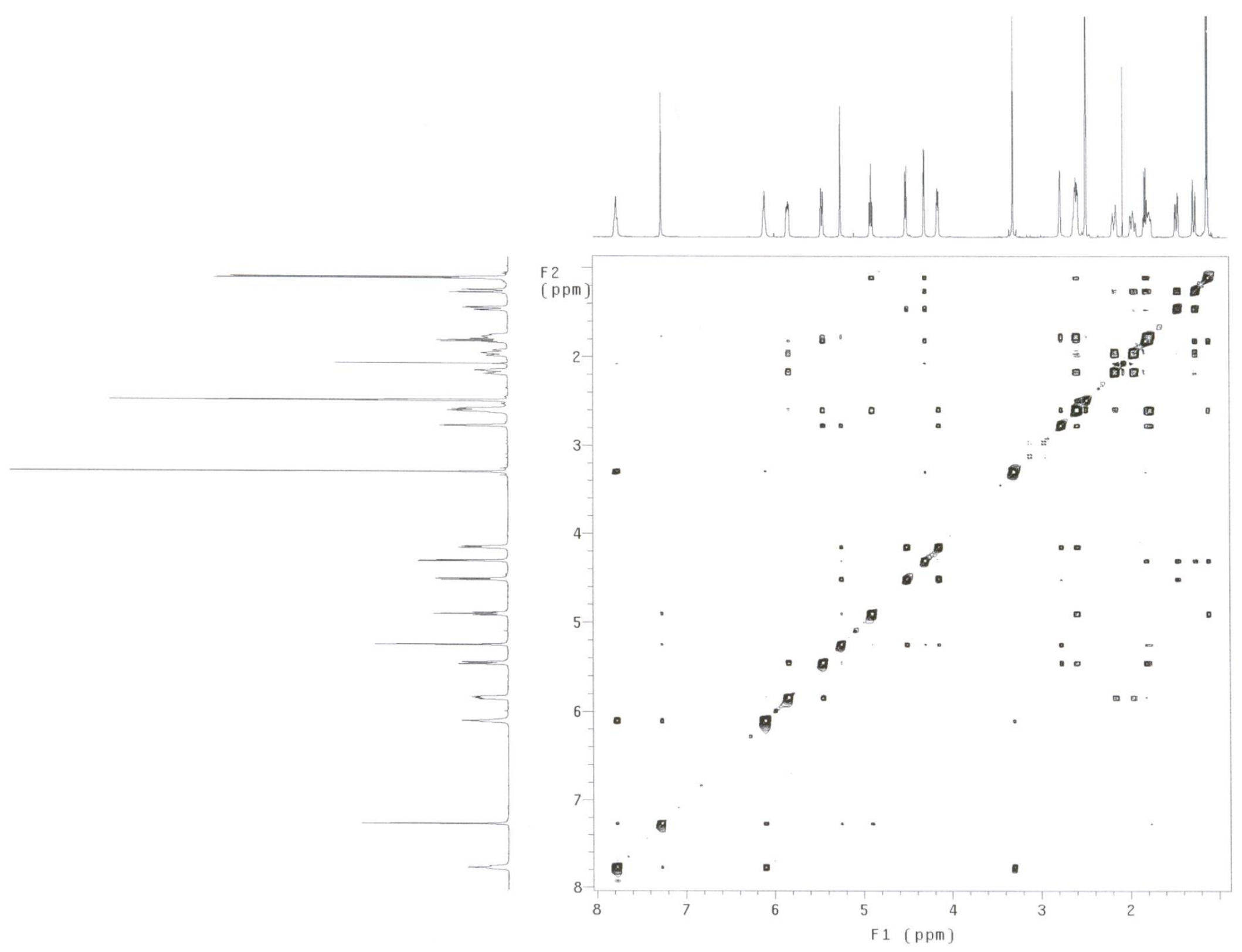

Figure S12. NOESY spectrum of compound $3\left(\right.$ DMSO-d $\left._{6}\right)$ 


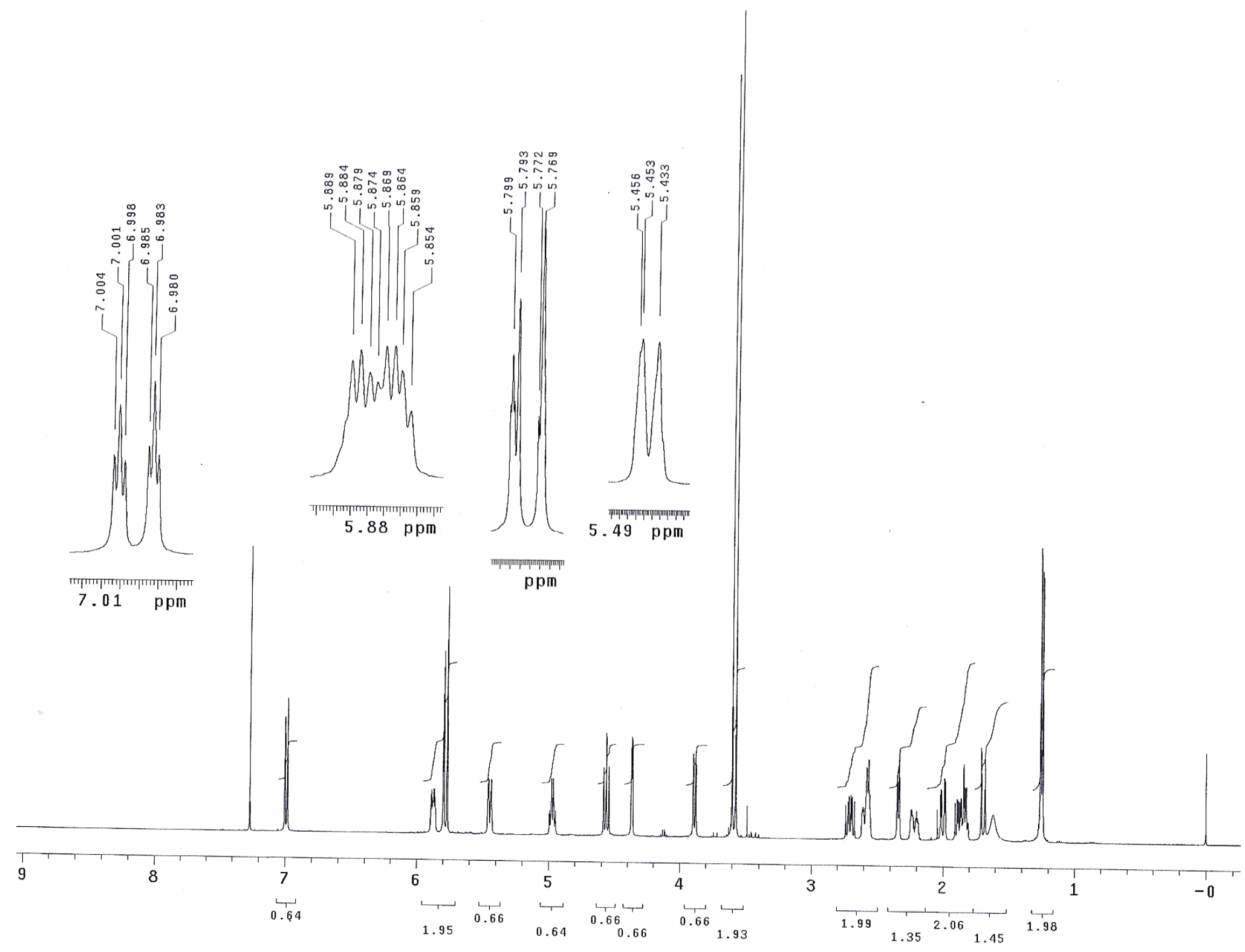

Figure S13. ${ }^{1} \mathrm{H}$ NMR spectrum of compound 4/5 $\left(\mathrm{CDCl}_{3}\right)$ 


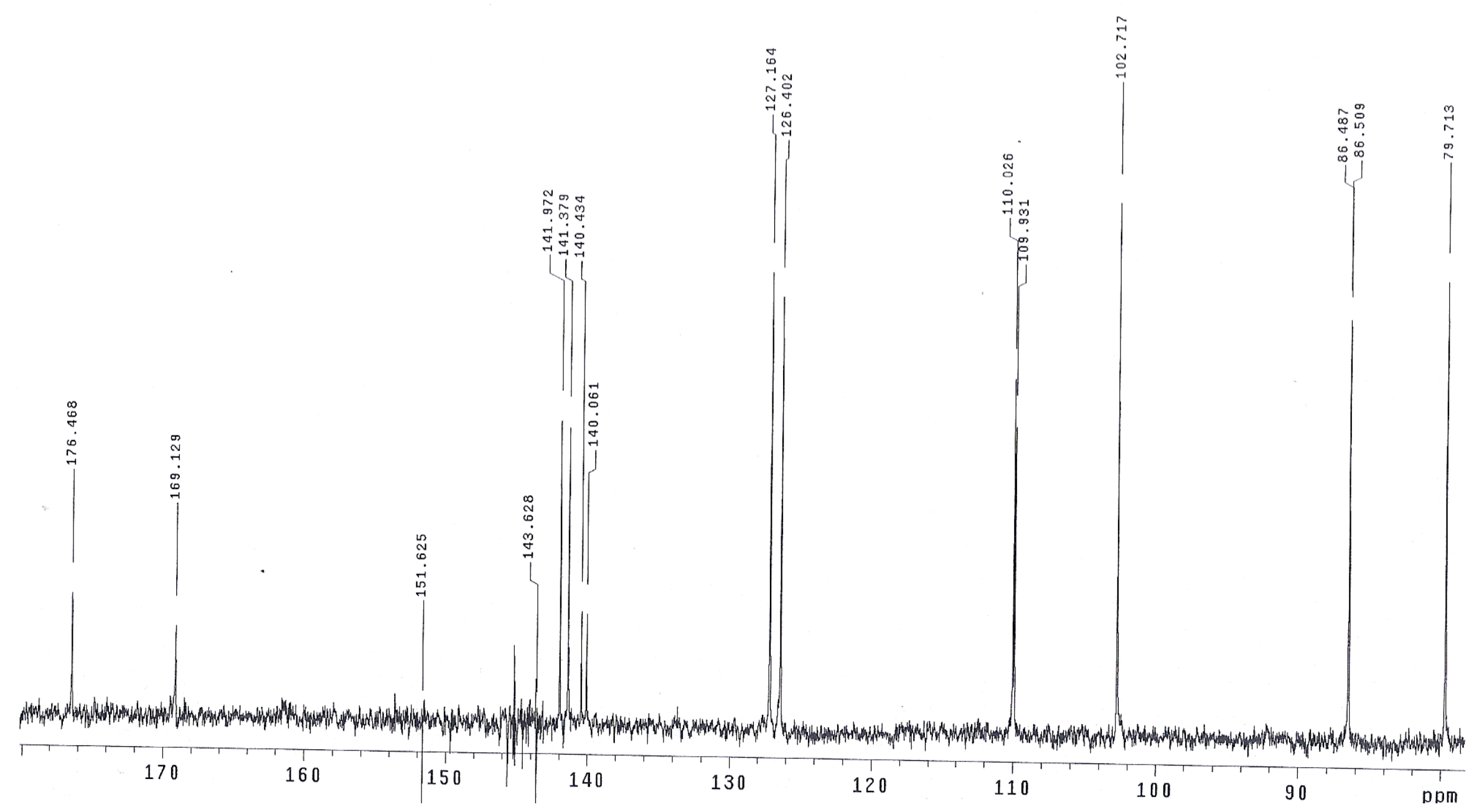

Figure S14. ${ }^{13} \mathrm{C}$ NMR spectrum of compound $\mathbf{4 / 5}\left(\mathrm{CDCl}_{3}\right)$ 


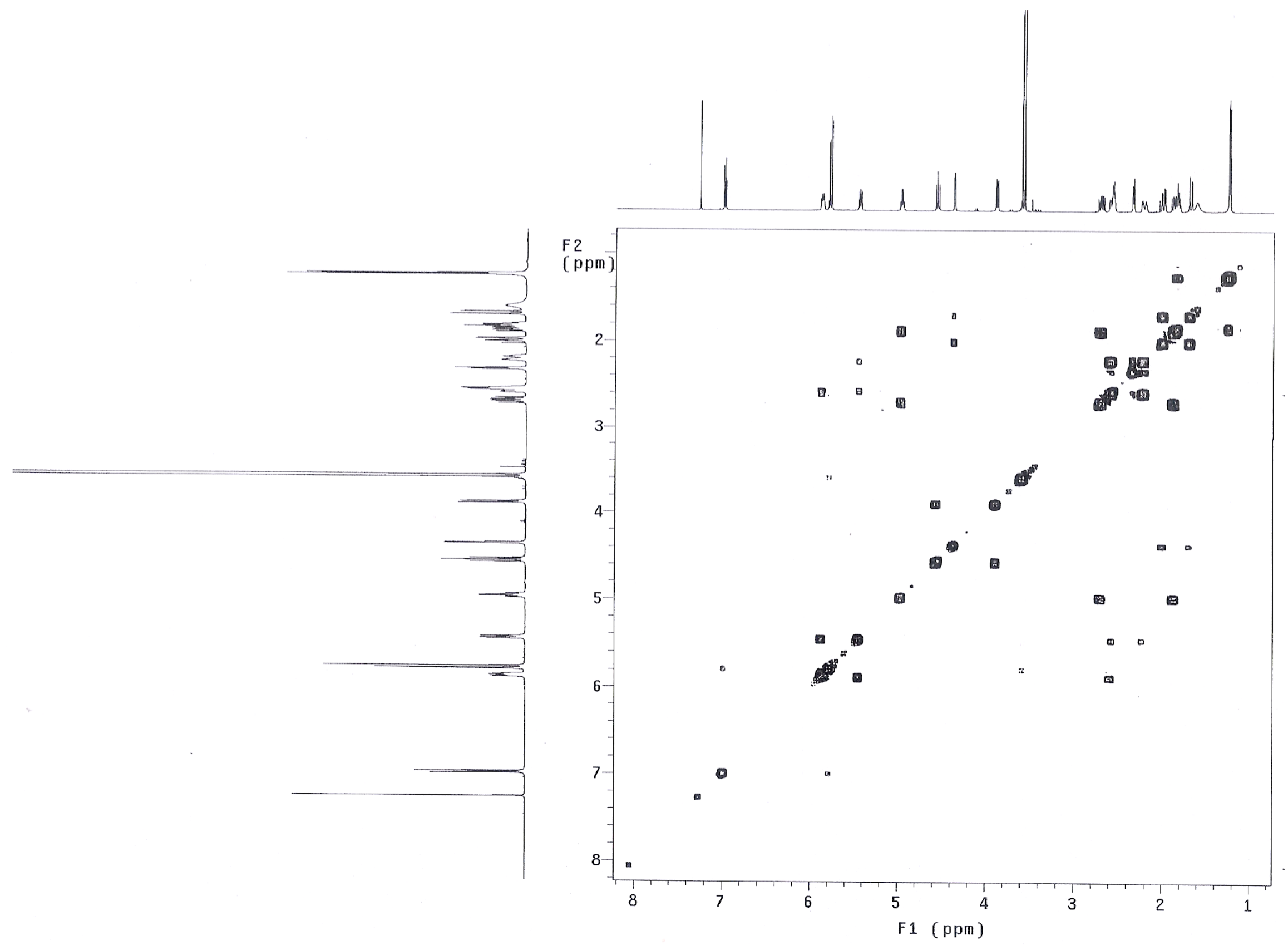

Figure S15. COSY spectrum of compound $\mathbf{4 / 5}\left(\mathrm{CDCl}_{3}\right)$ 


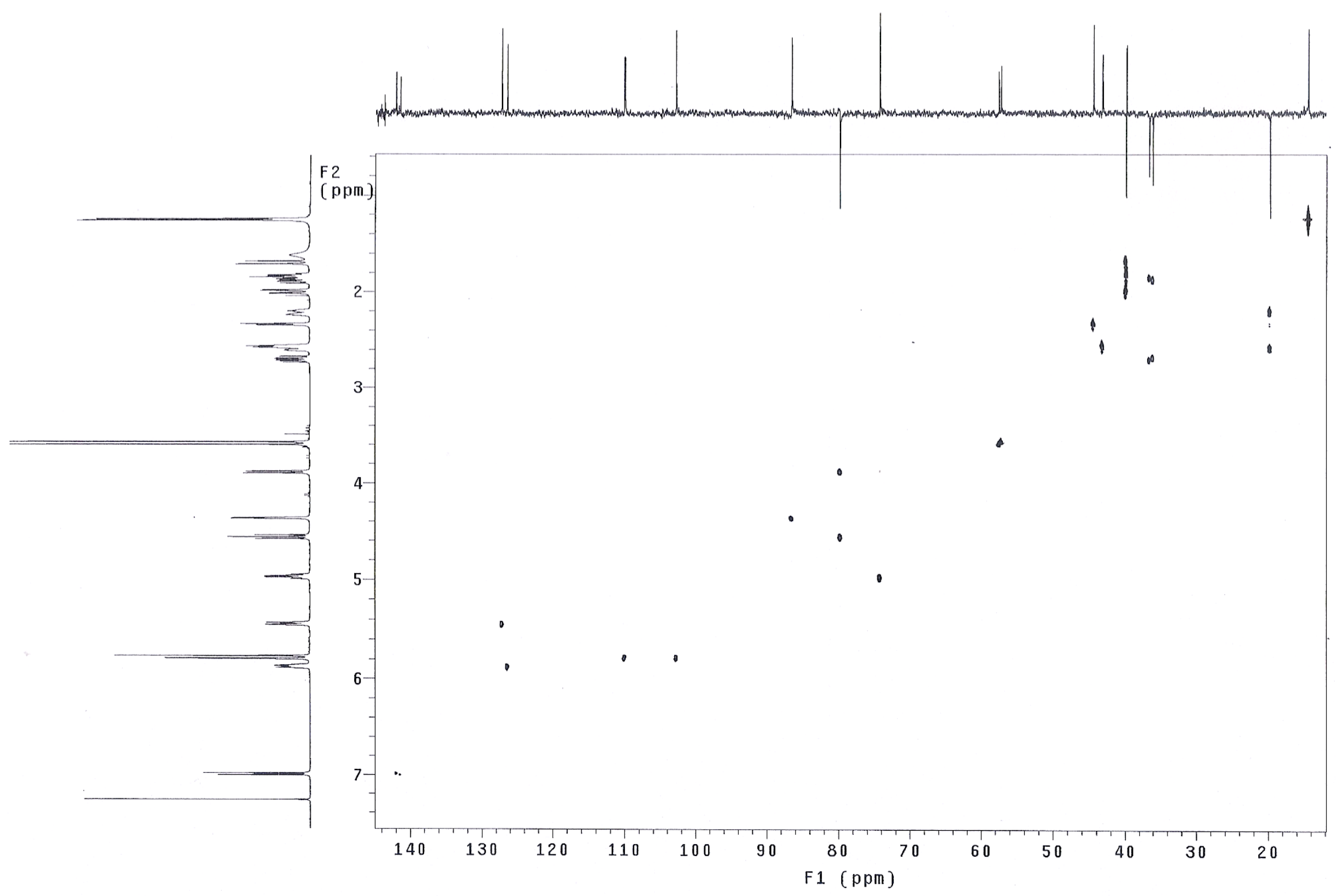

Figure S16. HSQC spectrum of compound $\mathbf{4} / \mathbf{5}\left(\mathrm{CDCl}_{3}\right)$ 


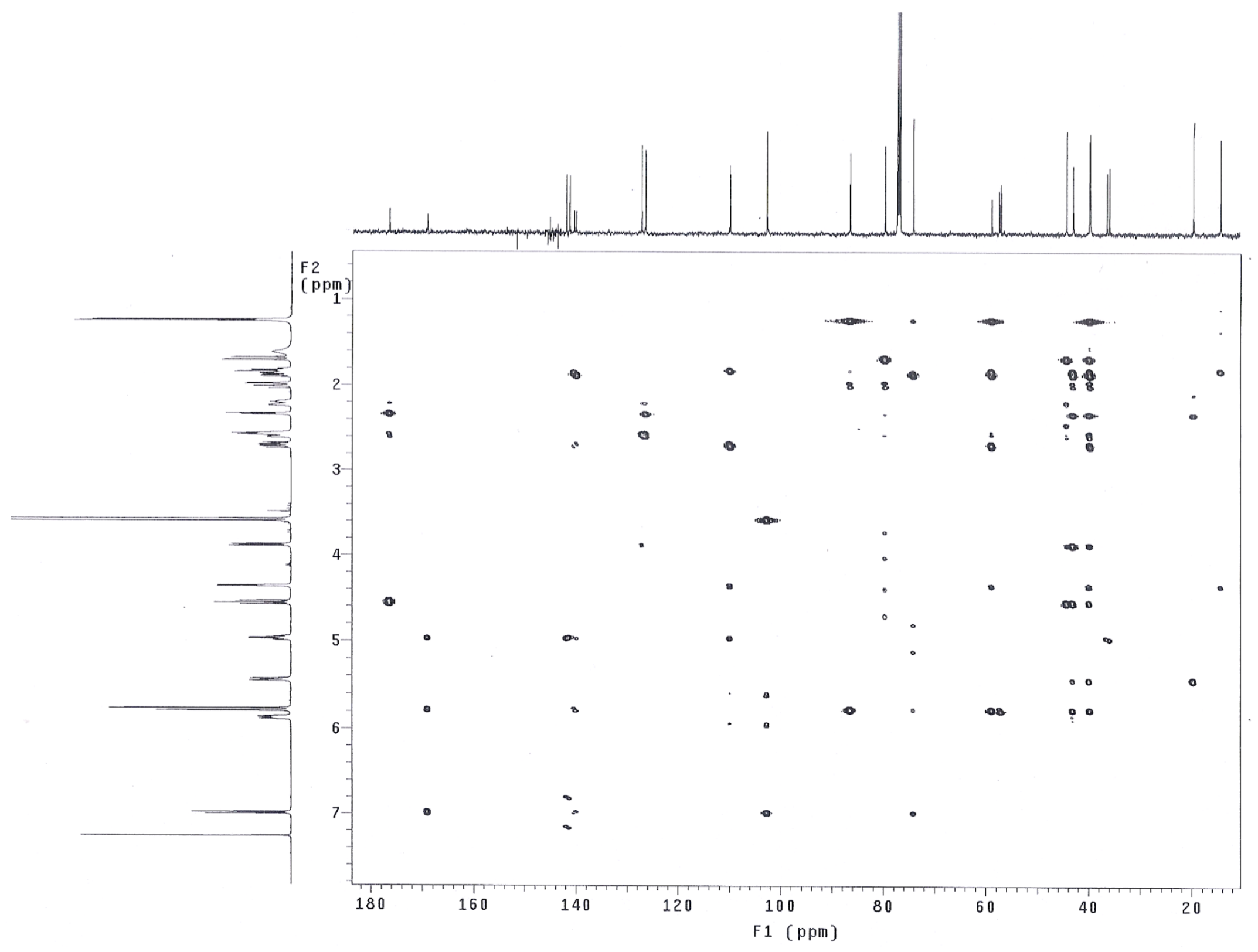

Figure S17. $\mathrm{HMBC}$ spectrum of compound $\mathbf{4 / 5}\left(\mathrm{CDCl}_{3}\right)$ 


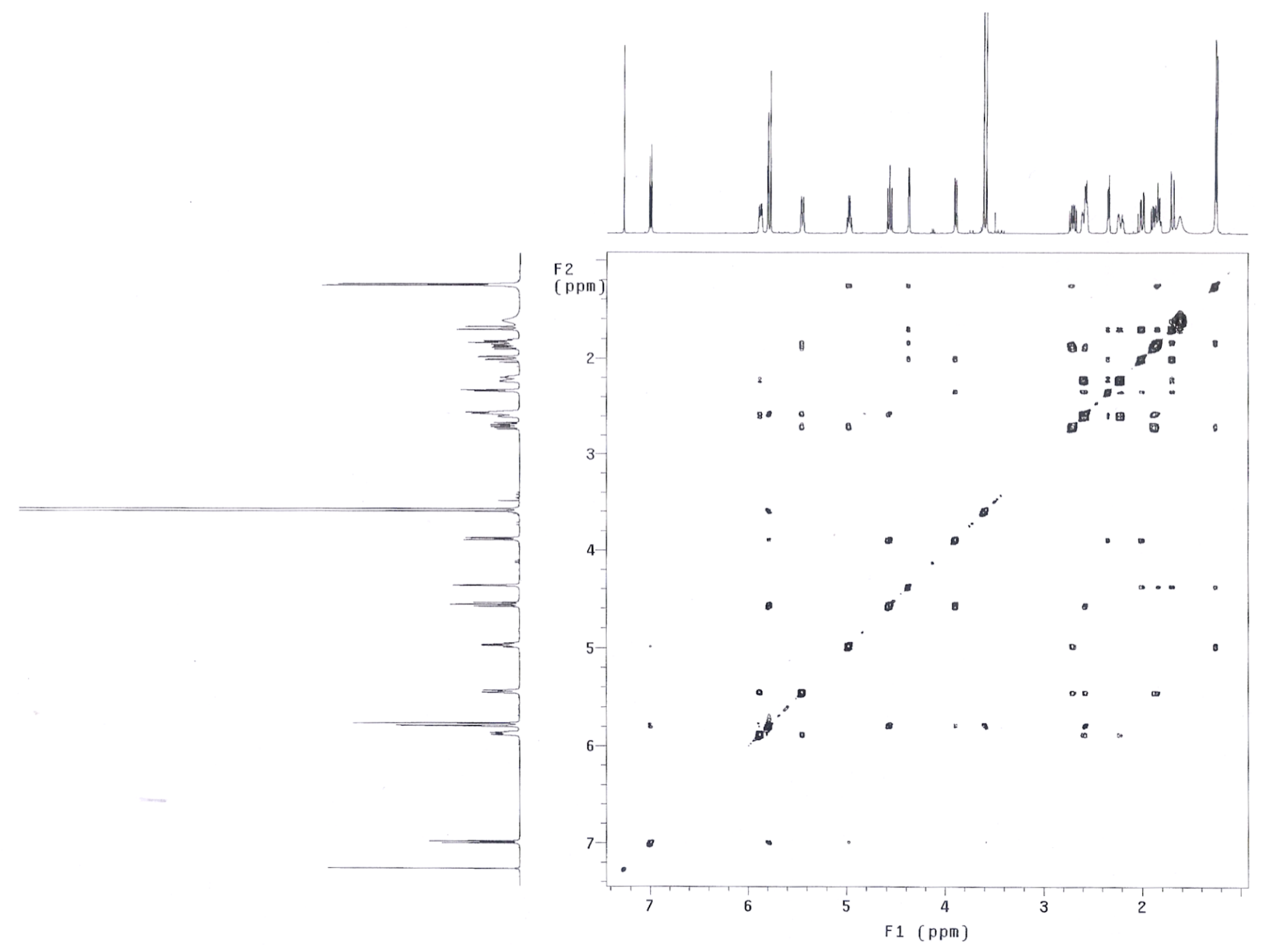

Figure S18. NOESY spectrum of compound $\mathbf{4 / 5}\left(\mathrm{CDCl}_{3}\right)$ 


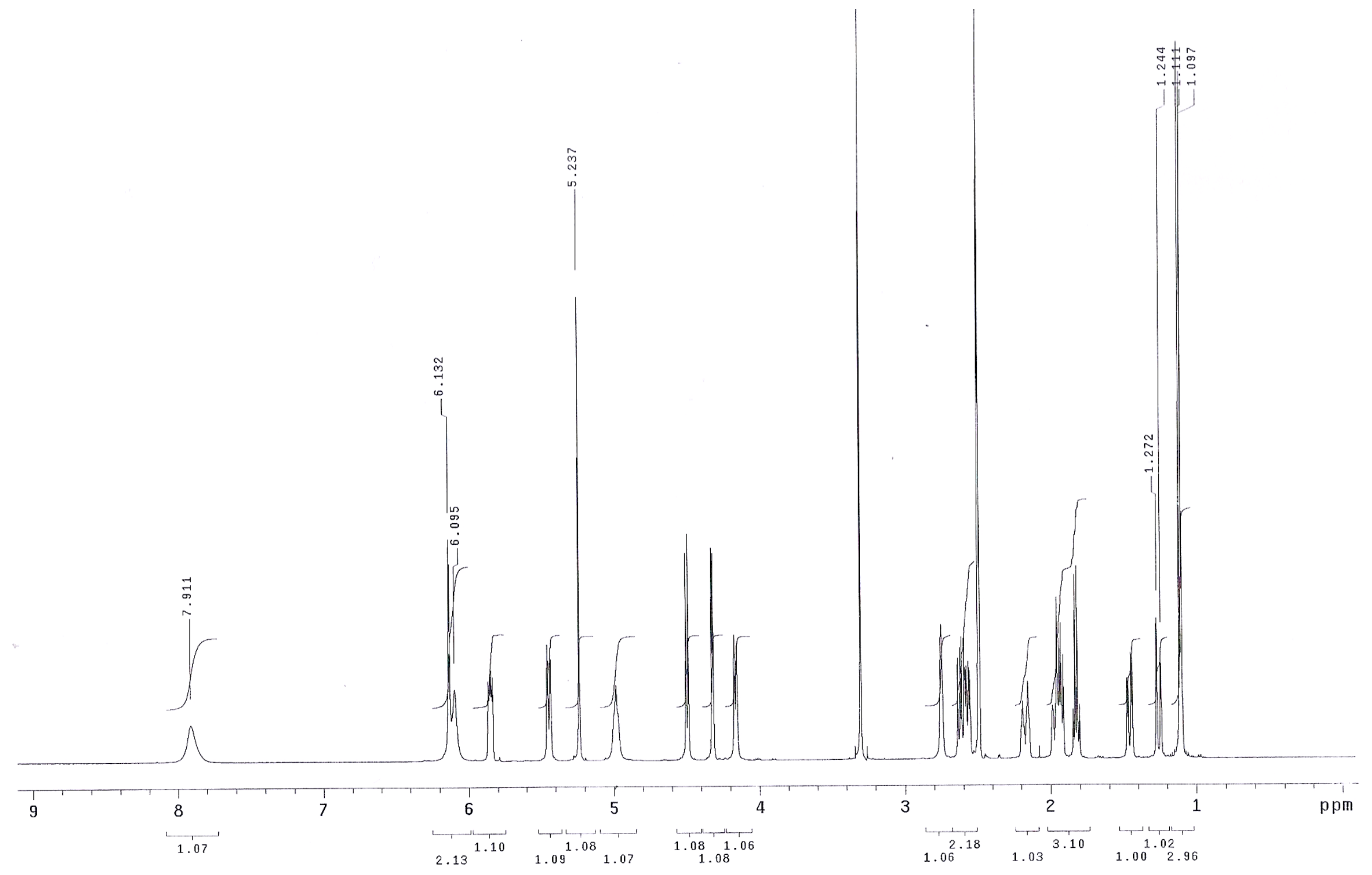

Figure S19. ${ }^{1} \mathrm{H}$ NMR spectrum of compound 7 (DMSO-d $\mathrm{d}_{6}$ ) 


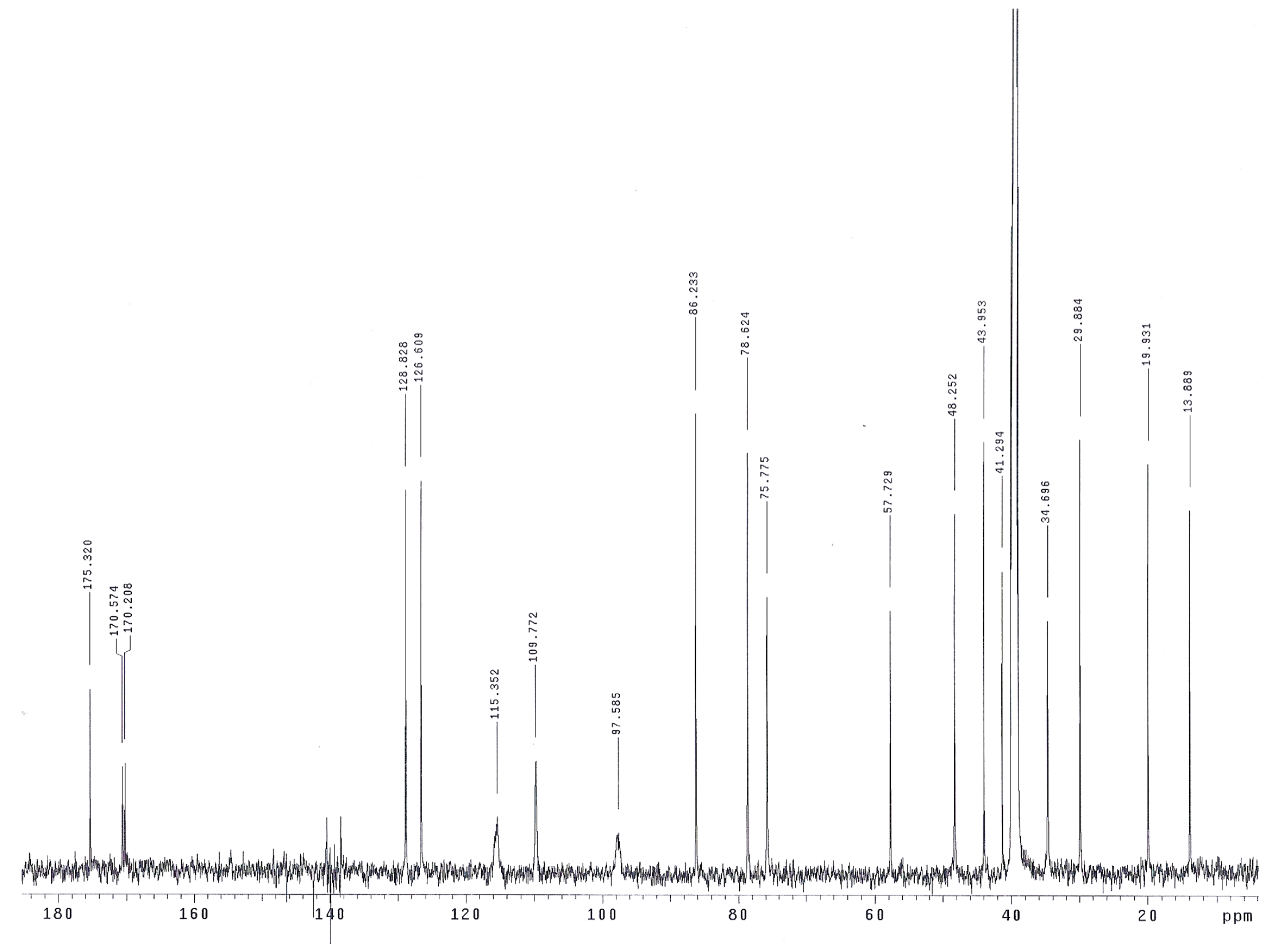

Figure S20 ${ }^{13} \mathrm{C}$ NMR spectrum of compound 7 (DMSO- $\mathrm{d}_{6}$ ) 


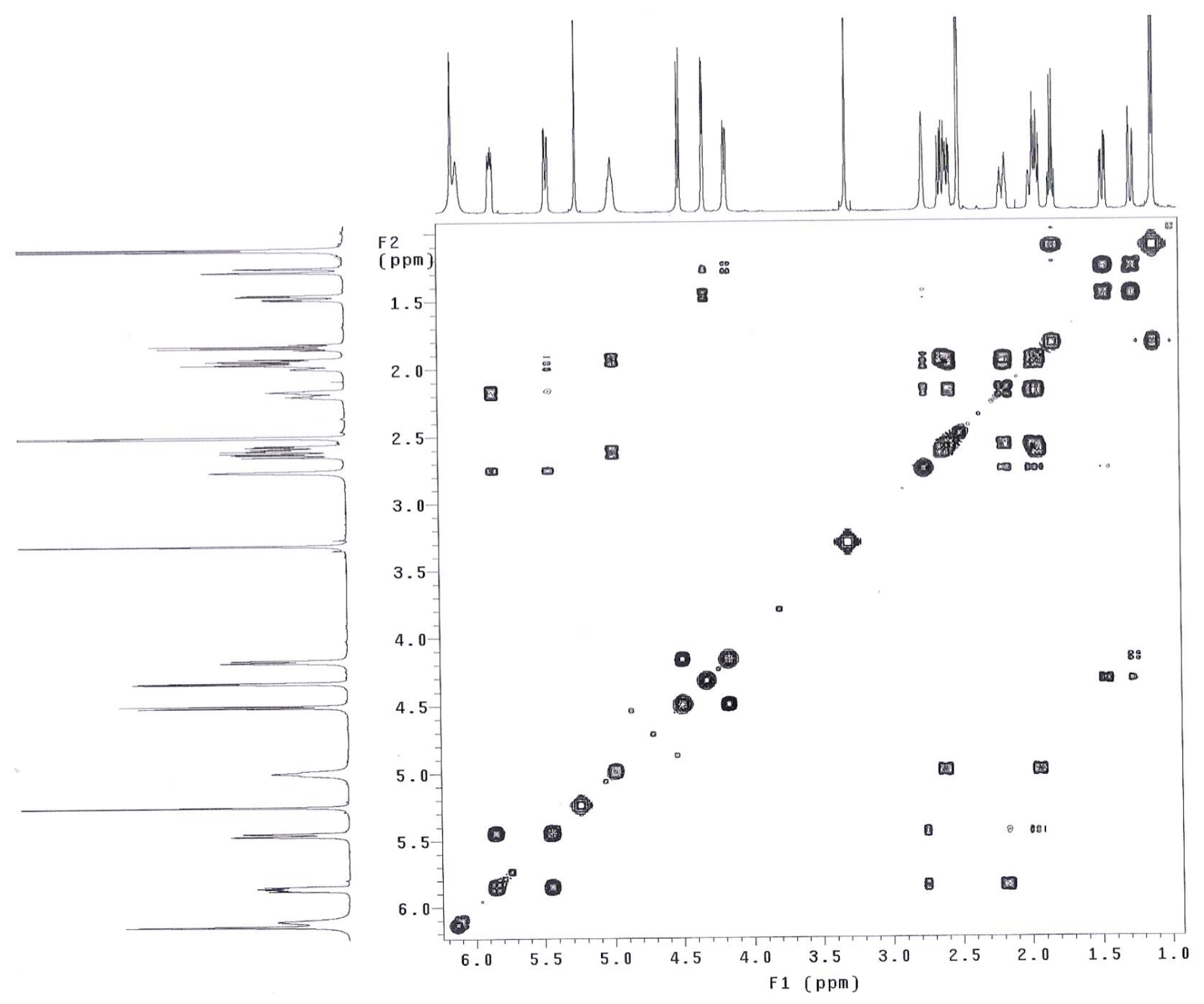

Figure S21. COSY spectrum of compound $7\left(\mathrm{CDCl}_{3}\right)$ 


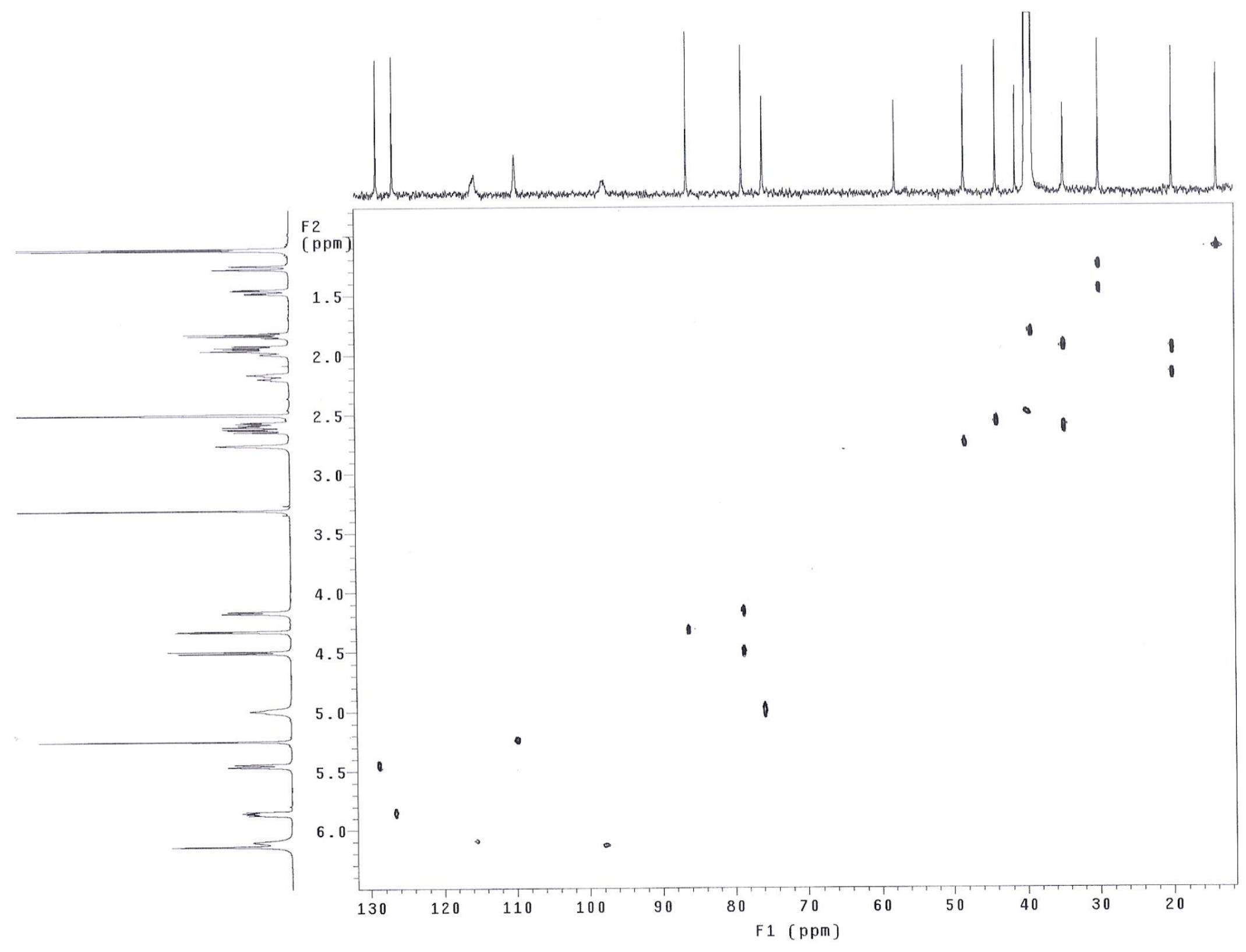

Figure S22. HSQC spectrum of compound 7 (DMSO-d ${ }_{6}$ ) 


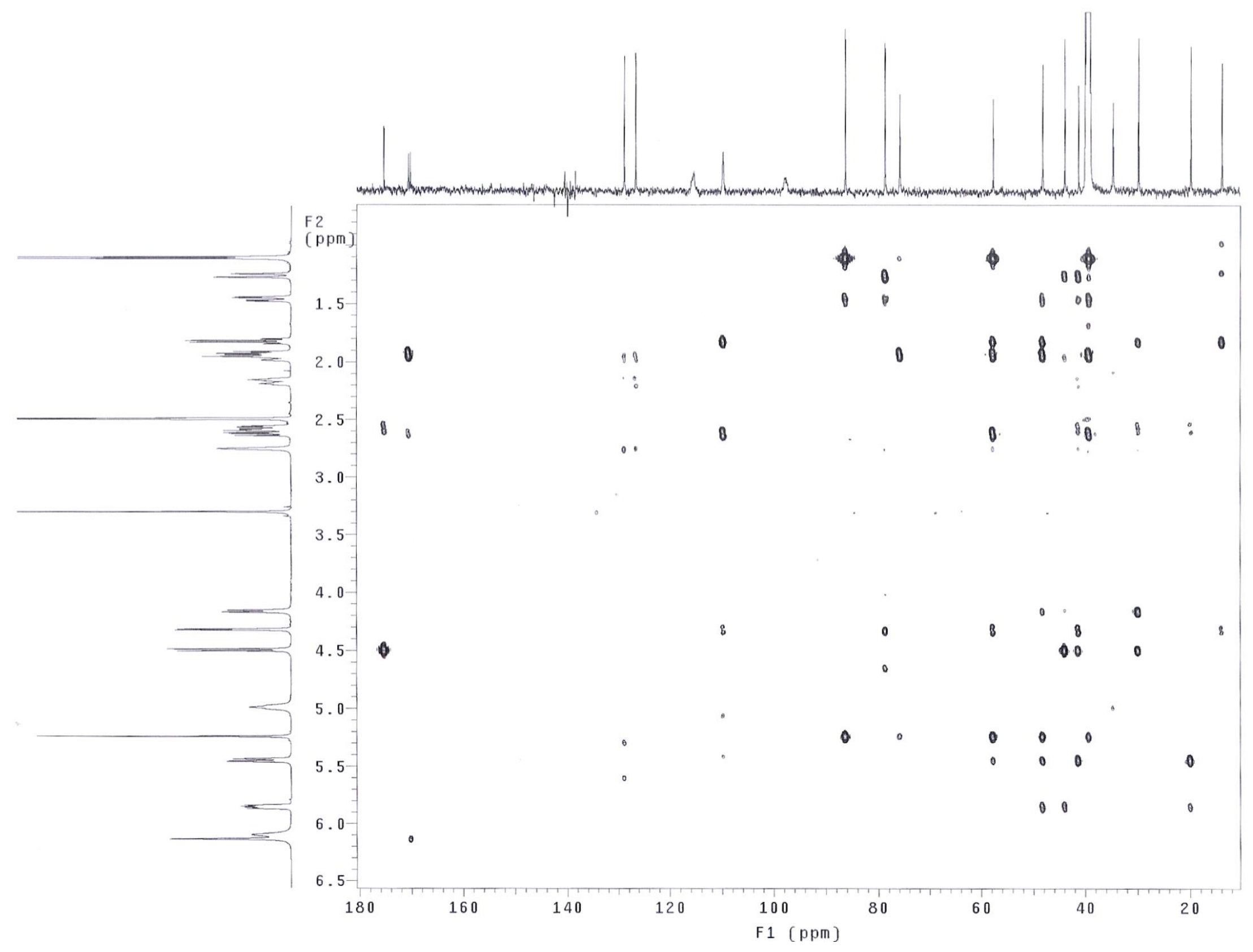

Figure S23. HMBC spectrum of compound 7 (DMSO-d $\left.\mathrm{D}_{6}\right)$ 


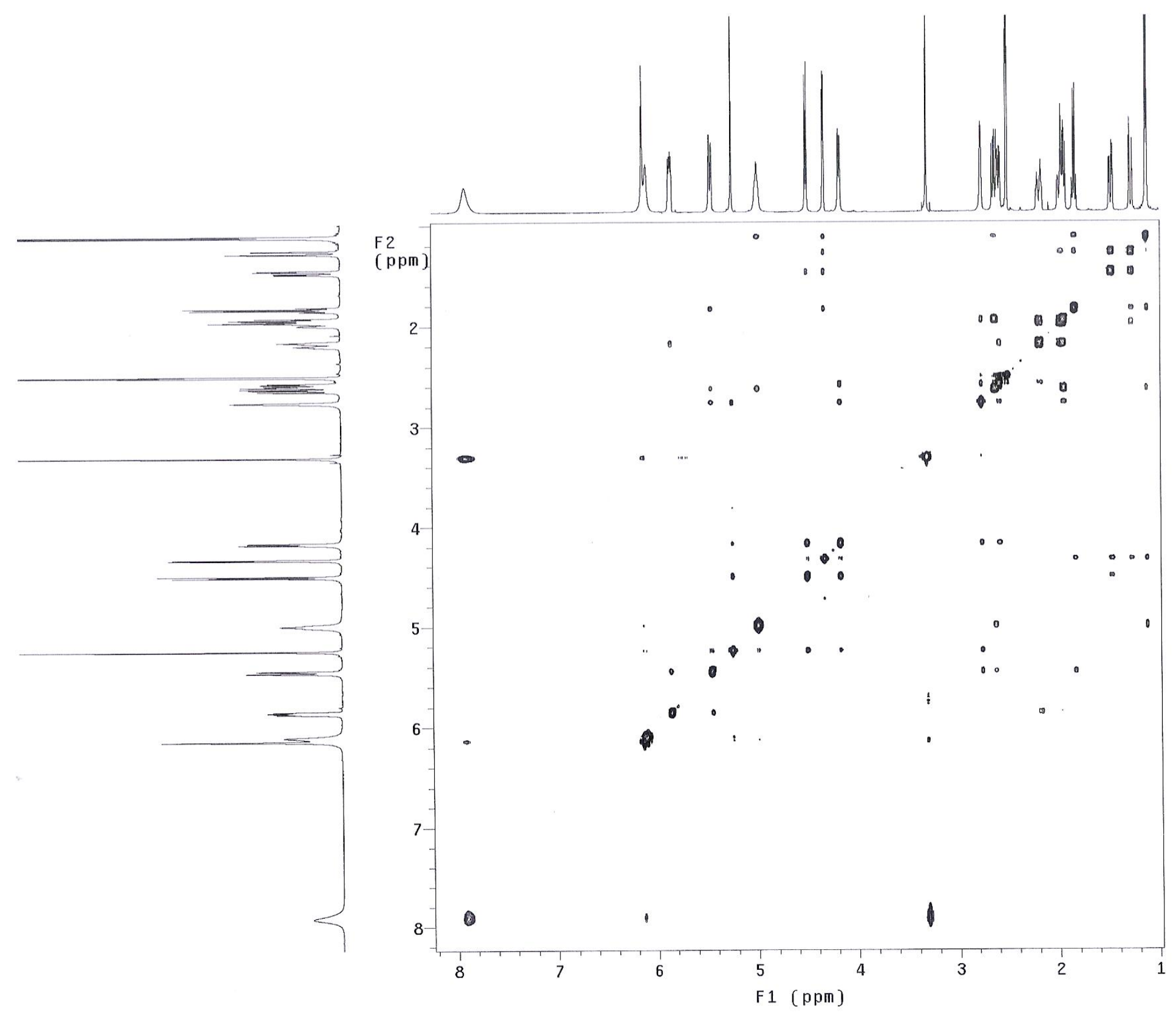

Figure S24. NOESY spectrum of compound 7 (DMSO-d $\left.{ }_{6}\right)$ 


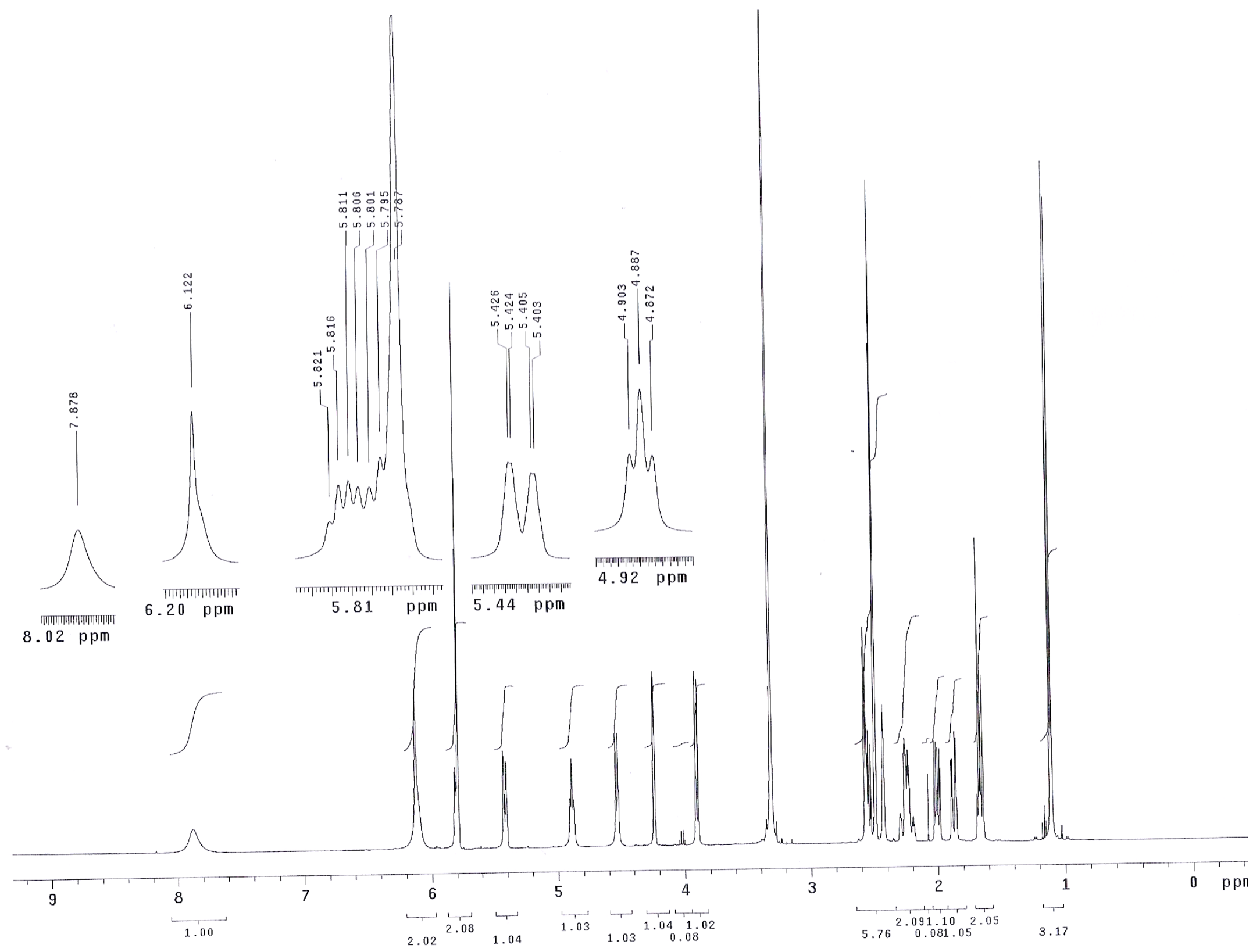

Figure S25. ${ }^{1} \mathrm{H}$ NMR spectrum of compound $8\left(\right.$ DMSO-d $\left._{6}\right)$ 


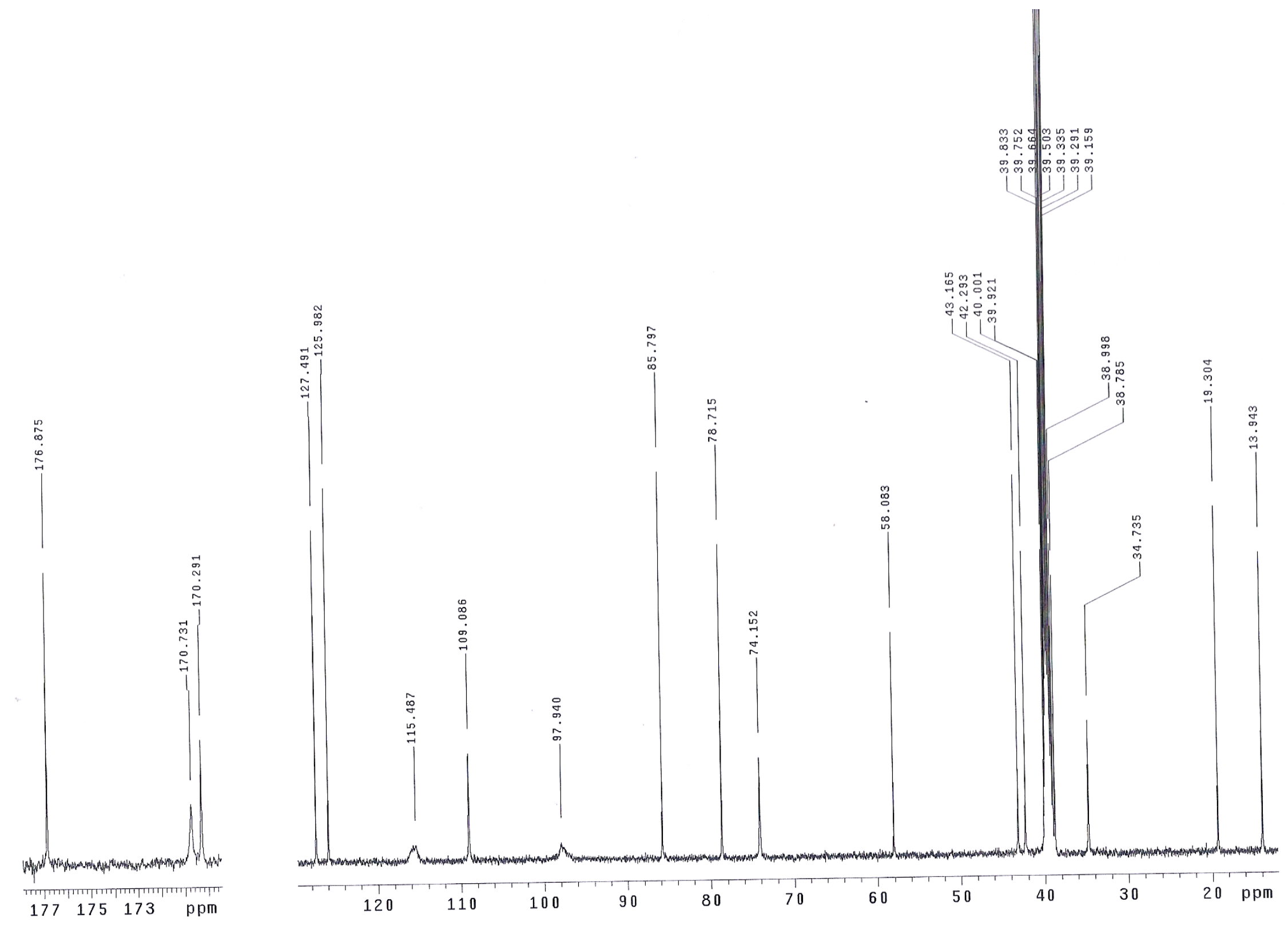

Figure S26 ${ }^{13} \mathrm{C}$ NMR spectrum of compound $8\left(\right.$ DMSO- $\left._{6}\right)$ 


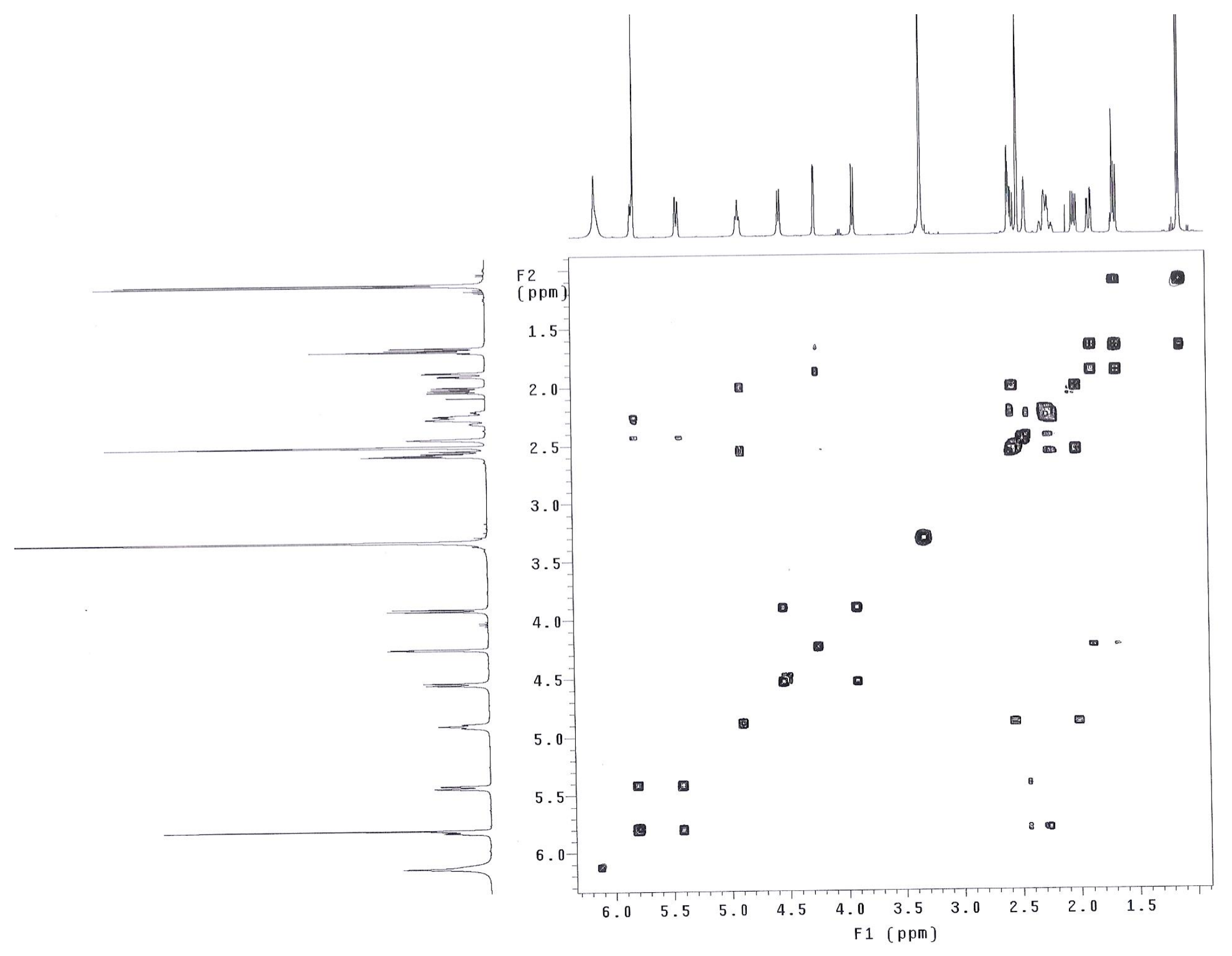

Figure S27. COSY spectrum of compound $8\left(\right.$ DMSO- $\left._{6}\right)$ 


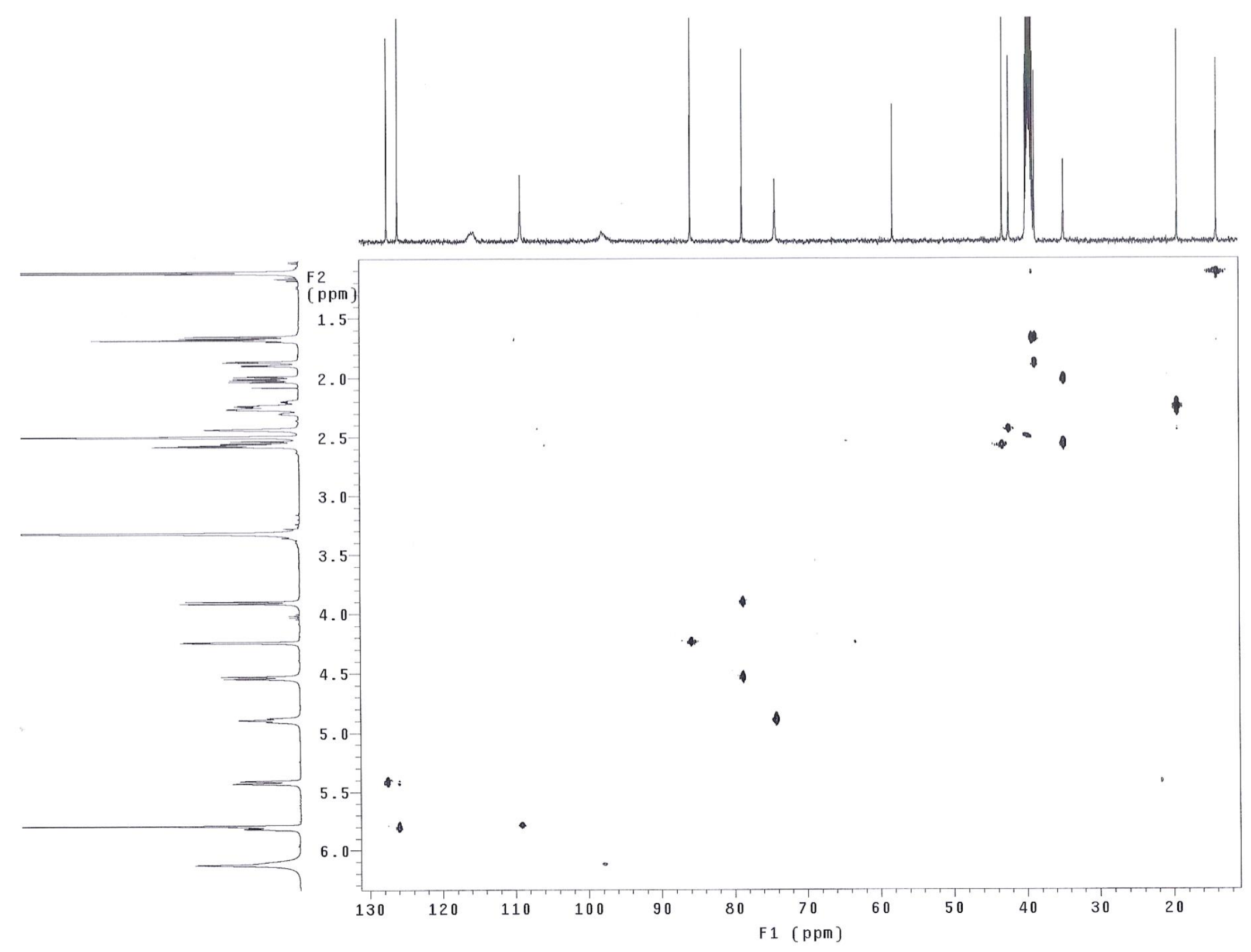

Figure S28. HSQC spectrum of compound $8\left(\mathrm{DMSO}^{-\mathrm{d}_{6}}\right)$ 


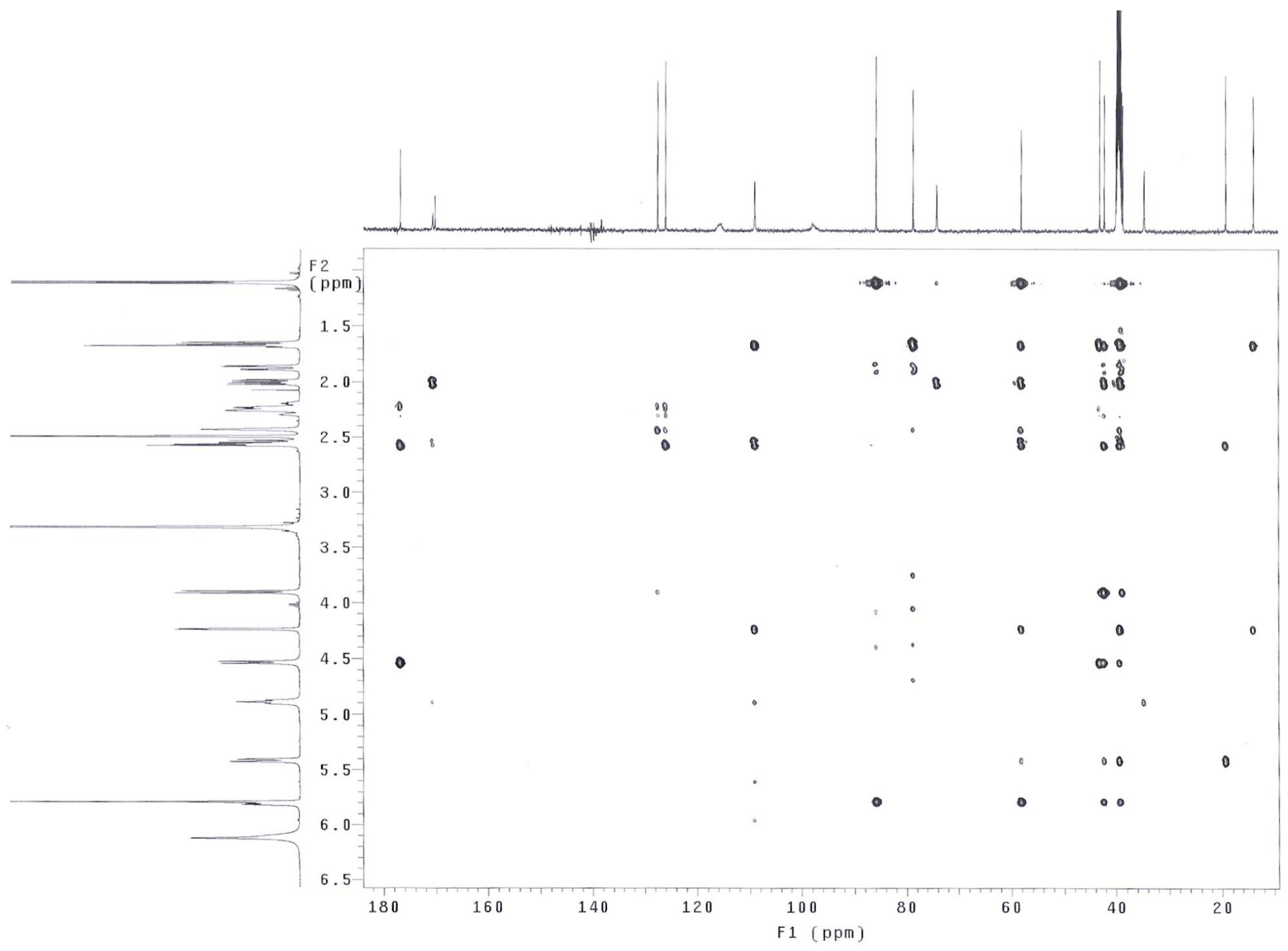

Figure S29. HMBC spectrum of compound 8 (DMSO- $\left.\mathrm{d}_{6}\right)$ 


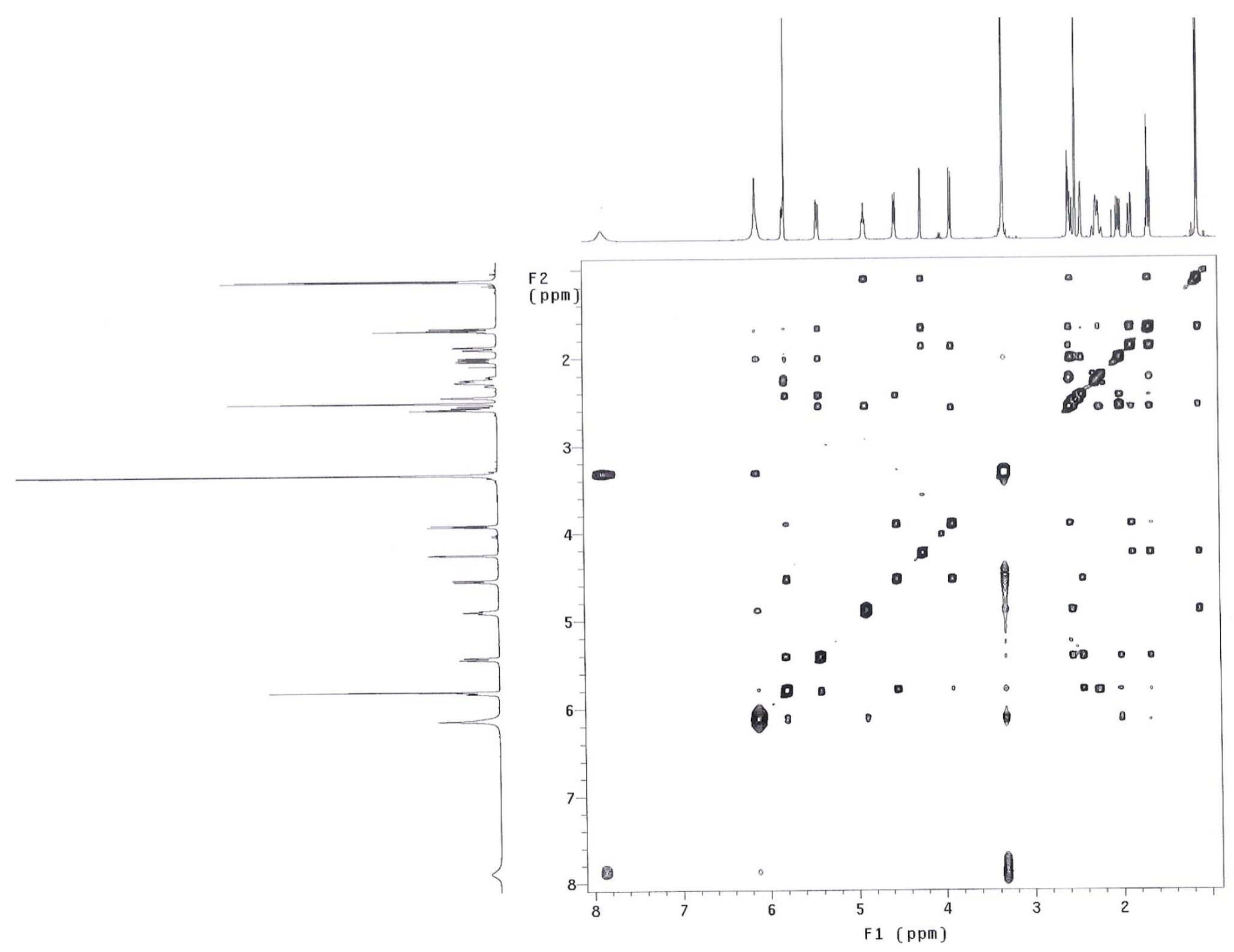

Figure S30. NOESY spectrum of compound $8\left(D M S O-d_{6}\right)$ 
Table S1. ${ }^{1} \mathrm{H}$ and ${ }^{13} \mathrm{C}$ NMR Data of Compound $10\left(500 / 125 \mathrm{MHz}, \mathrm{CDCl}_{3}\right)$.

\begin{tabular}{|c|c|c|c|c|c|}
\hline Position & ${ }^{1} \mathbf{H}$ & ${ }^{13} \mathrm{C}$ & Position & ${ }^{1} \mathbf{H}$ & ${ }^{13} \mathrm{C}$ \\
\hline 1 & 5.86 br d (12.0) & $126.9 \mathrm{CH}$ & 10 & 2.25 br s & $52.0 \mathrm{CH}$ \\
\hline 2 & $5.88 \mathrm{~m}$ & $129.0 \mathrm{CH}$ & $11 \alpha$ & $2.08 \mathrm{dd}(15.5,1.5)$ & $37.2 \mathrm{CH}_{2}$ \\
\hline 3 & $2.36 \mathrm{~m}$ & $21.3 \mathrm{CH}_{2}$ & $11 \beta$ & $2.74 \mathrm{dd}(15.5,8.0)$ & \\
\hline $3^{\prime}$ & $2.13 \mathrm{~m}$ & & 12 & 5.67 ddd $(8.0,1.5,1.5)$ & $70.6 \mathrm{CH}$ \\
\hline 4 & $2.38 \mathrm{dd}(13.0,6.0)$ & $46.4 \mathrm{CH}$ & 13 & & $127.3 \mathrm{C}$ \\
\hline 5 & & $41.9 \mathrm{C}$ & 14 & $6.40 \mathrm{~m}$ & $108.6 \mathrm{CH}$ \\
\hline $6 \alpha$ & $1.21 \mathrm{ddd}(14.0,3.0,3.0)$ & $18.2 \mathrm{CH}_{2}$ & 15 & $7.37 \mathrm{~m}$ & $144.3 \mathrm{CH}$ \\
\hline $6 \beta$ & 1.83 dddd $(14.0,14.0,4.5,2.0)$ & & 16 & $7.44 \mathrm{dd}(2.0,1.5)$ & $138.6 \mathrm{C}$ \\
\hline $7 \alpha$ & 1.68 dddd $(14.0,14.0,5.0,4.5)$ & $17.1 \mathrm{CH}_{2}$ & 17 & & $172.6 \mathrm{C}$ \\
\hline $7 \beta$ & $2.17 \mathrm{~m}$ & & 18 & & $175.7 \mathrm{C}$ \\
\hline 8 & $2.35 \mathrm{br} \mathrm{d}(4.5)$ & $41.2 \mathrm{CH}$ & $19_{\text {pro-S }}$ & $4.00 \mathrm{~d}(9.0,2.0)$ & $76.3 \mathrm{CH}_{2}$ \\
\hline \multirow[t]{2}{*}{9} & & $36.6 \mathrm{C}$ & $19_{\text {pro- } R}$ & $4.48 \mathrm{~d}(9.0)$ & \\
\hline & & & 20 & $1.26 \mathrm{~s}$ & 31.7 \\
\hline
\end{tabular}

